University of Nebraska - Lincoln

DigitalCommons@University of Nebraska - Lincoln

8-30-2012

\title{
X-ray and optical wave mixing
}

T. E. Glover

Lawrence Berkeley National Laboratory

D. M. Fritz

SLAC National Accelerator Laboratory

M. Cammarata

University Rennes, marco.cammarata@univ-rennes1.fr

T. K. Allison

University of Colorado

Sinisa Coh

University of California - Berkeley, sinisa@civet.berkeley.edu

See next page for additional authors

Follow this and additional works at: https://digitalcommons.unl.edu/physicsfuchs

Glover, T. E.; Fritz, D. M.; Cammarata, M.; Allison, T. K.; Coh, Sinisa; Feldkamp, J. M.; Lemke, H.; Zhu, D.; Feng, Y.; Coffee, Ryan; Fuchs, Matthias; Ghimire, S.; Chen, J.; Shwartz, S.; Reis, D. A.; Harris, S. E.; and Hastings, J. B., "X-ray and optical wave mixing" (2012). Matthias Fuchs Publications. 13.

https://digitalcommons.unl.edu/physicsfuchs/13

This Article is brought to you for free and open access by the Research Papers in Physics and Astronomy at DigitalCommons@University of Nebraska - Lincoln. It has been accepted for inclusion in Matthias Fuchs Publications by an authorized administrator of DigitalCommons@University of Nebraska - Lincoln. 


\section{Authors}

T. E. Glover, D. M. Fritz, M. Cammarata, T. K. Allison, Sinisa Coh, J. M. Feldkamp, H. Lemke, D. Zhu, Y. Feng, Ryan Coffee, Matthias Fuchs, S. Ghimire, J. Chen, S. Shwartz, D. A. Reis, S. E. Harris, and J. B. Hastings 


\title{
$X$-ray and optical wave mixing
}

\author{
T. E. Glover ${ }^{1}$, D. M. Fritz ${ }^{2}$, M. Cammarata ${ }^{3}$, T. K. Allison ${ }^{4}$, Sinisa Coh ${ }^{5,6}$, J. M. Feldkamp ${ }^{2}$, H. Lemke Lem $^{2}$ D. Zhu ${ }^{2}$, Y. Feng $^{2}$, \\ R. N. Coffee ${ }^{2}$, M. Fuchs ${ }^{7}$, S. Ghimire ${ }^{7}$, J. Chen ${ }^{7,8}$, S. Shwartz ${ }^{8}$, D. A. Reis ${ }^{7,8,9}$, S. E. Harris ${ }^{8,10}$ \& J. B. Hastings ${ }^{2}$
}

\begin{abstract}
Light-matter interactions are ubiquitous, and underpin a wide range of basic research fields and applied technologies. Although optical interactions have been intensively studied, their microscopic details are often poorly understood and have so far not been directly measurable. X-ray and optical wave mixing was proposed nearly half a century ago as an atomic-scale probe of optical interactions but has not yet been observed owing to a lack of sufficiently intense $\mathrm{X}$-ray sources. Here we use an X-ray laser to demonstrate X-ray and optical sum-frequency generation. The underlying nonlinearity is a reciprocal-space probe of the optically induced charges and associated microscopic fields that arise in an illuminated material. To within the experimental errors, the measured efficiency is consistent with first-principles calculations of microscopic optical polarization in diamond. The ability to probe optical interactions on the atomic scale offers new opportunities in both basic and applied areas of science.
\end{abstract}

Light-matter interactions have advanced our understanding of atoms, molecules and materials, and are also central to a number of areas of applied science. Although optical interactions have received a great deal of study, the microscopic details of how light manipulates matter are poorly understood in many circumstances. A material's optical response is complex, being determined by coupled many-body interactions that vary on the scale of atoms rather than on the scale of a long-wavelength applied field. Data are needed to combat this complexity, and so far it has not been possible to probe the microscopic details of light-matter interactions.

$\mathrm{X}$-ray and optical wave mixing, specifically sum-frequency generation (SFG), was proposed nearly half a century ago as an atomic-scale probe of light-matter interactions ${ }^{1,2}$. The process is, in essence, optically modulated X-ray diffraction: X-rays inelastically scatter from optically induced charge oscillations and probe optically polarized charge in direct analogy to how standard X-ray diffraction probes ground-state charge. Furthermore, the optically induced microscopic field is determined because it is closely related to the induced charge $e^{3-6}$. So far it has not been possible to measure these two quantities directly. $\mathrm{X}$-ray and optical wave mixing has frequently been discussed ${ }^{1,2,4,7-12}$, but it has not yet been demonstrated owing to a lack of sufficiently intense $\mathrm{X}$-ray sources. More generally, although there have been theoretical studies of nonlinear X-ray scattering ${ }^{13-18}$, experimental observations have largely been confined to the spontaneous processes of X-ray parametric down-conversion ${ }^{19-23}$ and resonant inelastic X-ray scattering ${ }^{24,25}$.

$\mathrm{X}$-ray free-electron lasers offer unprecedented brightness and new scientific opportunities ${ }^{26}$. Here we use an X-ray laser to demonstrate $\mathrm{X}$-ray/optical SFG through the nonlinear interaction of the two fields in single-crystal diamond. Optically modulated X-ray diffraction from the (111) planes generates a sum-frequency (X-ray plus optical) pulse. The measured conversion efficiency $\left(3 \times 10^{-7}\right)$ determines the (111) Fourier component of the optically induced charge and associated microscopic field that arise in the illuminated sample. To within experimental errors of $\sim 40 \%$, the measured charge density is consistent with first-principles calculations of microscopic optical polarization in diamond. The ability to measure atomic-scale charges and fields induced by light should contribute to a better understanding of materials and create new ways to study photo-triggered dynamics.

\section{$\mathrm{X}$-ray and optical wave mixing}

$\mathrm{X}$-ray and optical wave mixing is an atomic-scale probe of optical interactions: $\mathrm{X}$-rays provide atomic spatial resolution and light makes it possible selectively to probe optically polarized valence charge $e^{1,2}$ Sum-frequency radiation is produced when two applied fields simultaneously drive a coherent electronic response; the second field must polarize charge before the polarization due to the first field decays. For X-ray/optical SFG, the simultaneously polarized charge is equal to optically polarized valence charge because only this charge has significant polarizability for both the optical and X-ray fields. Tightly bound core electrons can only be polarized by the X-ray radiation. More generally, as the 'optical' wavelength is varied through the visible to the extreme-ultraviolet (EUV) and soft-X-ray regimes, the polarized charge corresponds to different charge components in a material. Therefore, various charge components can be selectively probed by adjusting the 'optical' wavelength. The SFG technique can, for instance, be extended to probe the full valence charge distribution by mixing X-rays with EUV radiation of a frequency high enough that all valence electrons respond uniformly as free electrons ${ }^{8,20,23}$ (but low enough that the polarizability of tightly bound core charge is negligible).

$\mathrm{X}$-ray/optical SFG is a parametric scattering process analogous to standard X-ray diffraction: kinematics are determined by energy and momentum conservation, and the generation of an SFG field is described by the wave equation. In direct analogy to standard X-ray crystallography, inelastically scattered sum-frequency X-rays probe a specific Fourier (that is, reciprocal-space) component of the charge density. Specifically, given an optical wavevector $k_{\mathrm{o}}$ and reciprocal lattice vector $G$, X-ray/optical SFG measures the $\left(Q=k_{\mathrm{o}}+G\right)$ th Fourier component of the optically induced change, $\delta \rho_{\mathrm{o}}(Q)$, to the valence charge density.

X-ray/optical SFG, and the closely related process of X-ray/optical difference-frequency generation, probes the linear optical susceptibility on a microscopic length scale. Higher-order X-ray/optical mixing 
processes can probe nonlinear optical susceptibilities. X-rays, for instance, could scatter from optically induced charge oscillating at twice the optical frequency and thereby be modified by two units of the optical photon energy; this would make it possible to study the atomic-scale details of optical second-harmonic generation.

\section{Observation of X-ray/optical SFG}

We choose diamond as the material in which to demonstrate X-ray/ optical SFG: its macroscopic optical ${ }^{27}$ and microscopic structural ${ }^{28}$ properties are well known and estimates exist for the X-ray/optical SFG efficiency in diamond, although these estimates differ by a factor of $\sim 100$ (refs $1,9,11)$. An X-ray pulse $(8,000 \mathrm{eV}, \sim 80 \mathrm{fs}$ ) and an optical pulse $\left(1.55 \mathrm{eV}, \sim 2 \mathrm{ps}, \sim 10^{10} \mathrm{~W} \mathrm{~cm}^{-2}\right)$ simultaneously illuminate a diamond sample slightly detuned from the optimum orientation for elastic Laue geometry diffraction from the (111) planes (Fig. 1a). Scattered X-rays are wavelength resolved using a $\mathrm{Si}(220)$ crystal. The combination of sample detuning and wavelength discrimination reduced the intensity of elastically scattered (background) light reaching our detector by seven to eight orders of magnitude relative to optimum elastic diffraction conditions.

An SFG signal was detected at the expected sample and analyser angles. The signal was observed to depend on the simultaneous presence of X-ray and optical beams, and on optimization we obtained a signal rate of $\sim 4,000$ detected photons per second. An energy analyser scan (Fig. 1b) confirms a detected photon energy equal to the sum of the input X-ray and optical photon energies and indicates a rockingcurve width $(17 \pm 2 \mu \mathrm{rad})$ in excellent agreement with the intrinsic analyser rocking-curve width $(\sim 17 \mu \mathrm{rad})$. This indicates that the SFG beam is spectrally narrow and well collimated compared with the energy spread $(\sim 310 \mathrm{meV})$ and angle spread $(\sim 17 \mu \mathrm{rad})$ transmitted by the analyser (called the analyser acceptance). Simulations discussed below are in agreement with this observation.

The analyser was fixed in position and the variation of SFG power with sample angle was measured (Fig. 1c). The signal is sharply peaked at the angle satisfying energy and momentum conservation, and the measured rocking-curve width $(8.1 \pm 0.7 \mu \mathrm{rad})$ is equal, to within the errors, to half the measured analyser width $(17 \pm 2 \mu \mathrm{rad})$. A sample rotation of $\delta \theta$ causes a $2 \delta \theta$ rotation of the output beam, which indicates that the sample rocking-curve width is set by the limited energy and angle acceptance of the analyser. This observation supports the above conclusion obtained from consideration of the analyser rocking curve (Fig. 1b), namely that the SFG beam is narrow in energy and angle compared with the analyser acceptance. These observations place a lower limit of $\sim 8 \mu \mathrm{rad}$ on the SFG rocking-curve width because the SFG intensity is constant over an angular range $(\sim 8 \mu \mathrm{rad})$ limited by the finite analyser acceptance. Simulations discussed below predict an SFG rocking-curve width of $20 \mu \mathrm{rad}$.

Figure 1d shows how the SFG signal varies with rotation of the optical polarization vector. We expect the efficiency to vary as $\left(\varepsilon_{\mathrm{o}} \bullet G_{111}\right)^{2}$, where $\varepsilon_{\mathrm{o}}$ is the optical polarization vector and $G_{111}$ is the (111) lattice vector. This scaling reflects the quadratic dependence of SFG efficiency on the linearly induced optical charge, which in turn varies as $\varepsilon_{\mathrm{o}} \bullet G_{111}$. The SFG signal exhibits the expected dependence: it is maximized when $\varepsilon_{\mathrm{o}}$ is in the scattering plane and it is zero when $\varepsilon_{\mathrm{o}}$ is normal to the scattering plane.

The SFG efficiency was determined by measuring the incident $\mathrm{X}$-ray pulse energy (photons per pulse) and, under both elastic Laue and SFG conditions, the outgoing X-ray pulse energy. The measured
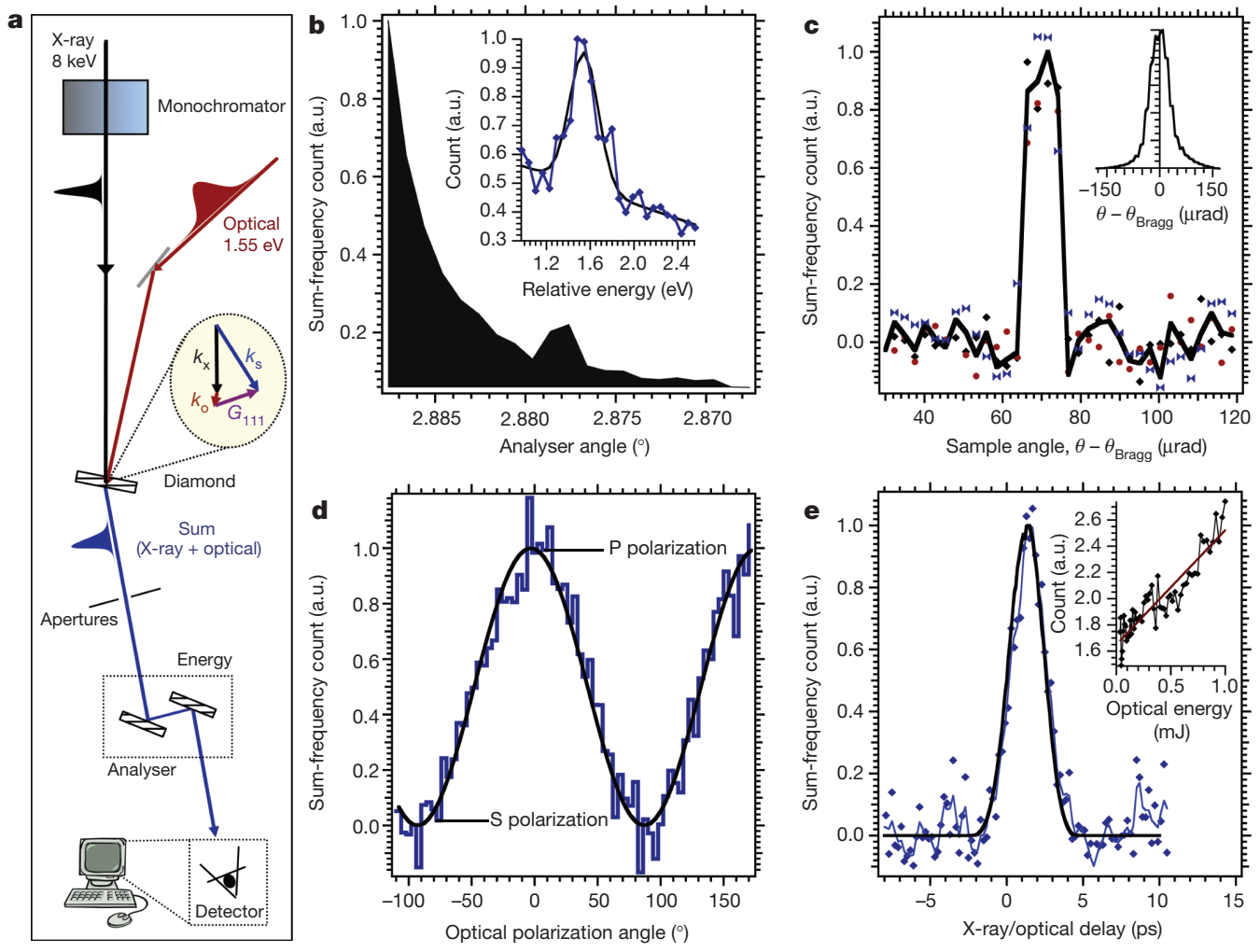

Figure $1 \mid$ X-ray/optical SFG experiment. a, Experimental layout for generation of X-ray (8-keV) and optical (1.55-eV) SFG in diamond. a.u., arbitrary units. b, SFG signal versus energy analyser angle. Inset, energy relative to $8 \mathrm{keV}$. The black line is a Gaussian fit $(310 \pm 35 \mathrm{meV}, 17 \pm 2-\mu \mathrm{rad}$ full-width at half-maximum). The SFG energy exceeds the X-ray energy by one optical photon. c, SFG signal versus diamond-sample angle: average of three data scans (width, $\sim 8 \mu \mathrm{rad}$; black line) and individual scans (markers). Energy and momentum conservation is satisfied at $\theta-\theta_{\mathrm{Bragg}} \approx 70 \mu \mathrm{rad}$. Inset, sample rocking curve for elastic diffraction (width, $\sim 65 \mu \mathrm{rad}$ ). d, SFG signal (measured, blue; expected variation, black) versus angle of optical polarization vector $\left(\varepsilon_{\mathrm{o}}\right)$. The signal is maximum when $\varepsilon_{\mathrm{o}}$ is in the diffraction plane $\left(0^{\circ}\right)$ and is zero when $\varepsilon_{\mathrm{o}}$ is normal to the diffraction plane $\left( \pm 90^{\circ}\right)$. e, SFG signal versus $\mathrm{X}$-ray/optical time delay (raw data, blue markers; one-point-smoothed data, blue curve). Black cross-correlation curve (2.5-ps full-width at half-maximum): solution of wave equation for an 80-fs X-ray pulse and a 1.7-ps optical pulse. Inset, SFG signal versus optical intensity. The red line is a fit to a linear dependence on optical intensity. 
efficiency is $3 \times 10^{-7}$ and the estimated uncertainty is a multiplicative factor of two. The SFG signal strength is a bilinear function of the input beam intensities, varying as the product of the optical and input $\mathrm{X}$-ray intensities. Because the efficiency is defined as the number of SFG photons per input X-ray photon, it is independent of input X-ray intensity but is linearly dependent on optical intensity.

Finally, an X-ray/optical cross-correlation is demonstrated. To within errors of $\sim 50 \%$, the SFG signal is observed to vary linearly with optical intensity (Fig. 1e, inset). The measured variation in the SFG signal with X-ray/optical relative time delay indicates a crosscorrelation width of $\sim 2.5$ ps (Fig. 1e). By solving the wave equation, we compute the SFG intensity as a function of relative time delay to determine that the measured cross-correlation is reproduced by a simulation with an 80 -fs X-ray pulse and a 1.7-ps optical pulse, the second of which is consistent with the experimental optical pulse duration $(2 \pm 0.5 \mathrm{ps})$. The simulated cross-correlation is also shown in Fig. 1e. The cross-correlation width is set by two factors: the duration of the (long) optical pulse and the fact that the X-ray and optical pulses move through the material at different speeds and in different directions.

\section{Wave equation model}

The generation of a sum-frequency electric field is described by the time- and space-dependent wave equation. The slowly-varyingenvelope approximation leads to the following equation relating the sum-frequency field $\left(E_{\mathrm{s}}\right)$ and bilinear current $\left(J_{\mathrm{NL}}\right)$ envelopes (Supplementary Information, section 3):

$$
\cos \left(\theta_{\mathrm{s}}\right) \frac{\mathrm{d} E_{\mathrm{s}}}{\mathrm{d} z}+\sin \left(\theta_{\mathrm{s}}\right) \frac{\mathrm{d} E_{\mathrm{s}}}{\mathrm{d} x}+\frac{1}{c} \frac{\mathrm{d} E_{\mathrm{s}}}{\mathrm{d} t}+\beta E_{\mathrm{s}}=-\frac{2 \pi}{c} J_{\mathrm{NL}}
$$

The $z$ and $x$ axes are respectively the inward-facing surface normal and an arbitrary line parallel to the sample surface, $\beta$ is the X-ray absorption coefficient and $\theta_{\mathrm{s}}$ is the angle between the $z$ axis and the sum-frequency wavevector.

$\mathrm{X}$-rays see diamond as a periodic collection of free electrons $\left(h v_{\mathrm{x}}\right.$ $\gg h v_{\text {binding, }}$, where $h v_{\mathrm{x}}$ is the X-ray photon energy and $h v_{\text {binding }}$ is the electron binding energy; $h$ is Planck's constant), and the bilinear current, the source of $E_{\mathrm{s}}$, is written as a charge times a velocity ${ }^{29}$. We find that the dominant current is the 'Doppler' current
(Supplementary Information, section 4), which represents inelastic scatter as X-rays $\left(E_{\mathrm{x}}\right)$ impart a first-order velocity to an optically induced charge density $\left(\delta \rho_{\mathrm{o}}\right)$ :

$$
\begin{array}{r}
J_{\mathrm{NL}}\left(k_{\text {sum }}=Q+k_{\mathrm{x}}, \omega_{\text {sum }}=\omega_{\mathrm{o}}+\omega_{\mathrm{x}}\right) \\
=\frac{e}{i m \omega_{\mathrm{x}}} \delta \rho_{\mathrm{o}}\left(Q, \omega_{\mathrm{o}}\right) \varepsilon_{\mathrm{s}} \bullet E_{\mathrm{x}}\left(k_{\mathrm{x}}, \omega_{\mathrm{x}}\right)
\end{array}
$$

In equation (2), $\varepsilon_{\mathrm{s}}$ is the sum-frequency polarization vector, $e$ and $m$ are respectively the electron charge and mass, $k_{\mathrm{x}}$ and $\omega_{\mathrm{x}}$ are respectively the X-ray wavevector and frequency, and $\delta \rho_{\mathrm{o}}\left(Q, \omega_{\mathrm{o}}\right)$ is the Fourier component of the optically induced charge oscillating with spatial wavevector $Q=k_{\mathrm{o}}+G_{111}$ and optical frequency $\omega_{\mathrm{o}}$; it is induced by, and is therefore a function of, the external applied optical field, $E_{\text {light }}$. The input pulses $E_{\mathrm{x}}$ and $E_{\text {light }}$ are modelled as Gaussians in space and time with linear chirp parameters determined by their angle-energy spread. With $J_{\mathrm{NL}}$ as given in equation (2) as the source term, we solve the equation obtained by taking a two-dimensional Fourier transform of equation (1).

The power distributions in angle-energy space for the calculated SFG and the driving bilinear current power distributions are shown in Fig. $2 \mathrm{a}-\mathrm{c}$ for crystal lengths of 1,10 and $500 \mu \mathrm{m}$ (the last of which corresponds to our sample). The induced current, which is proportional to the space-time product of the input beams, has an angle-energy power distribution determined by convolution of the Fourier-space input-beam profiles. The angle-energy width of the bilinear current $(\sim 4 \mu \mathrm{rad}, \sim 1 \mathrm{eV})$ is determined primarily by the angle-energy width of the input X-ray beam; its energy spread $(1 \mathrm{eV})$ is large compared with that of the optical beam $(<0.1 \mathrm{eV})$, and its transverse wavevector spread is approximately twice that of the optical beam. The angle-energy width of the weakly scattered SFG beam is a fraction of the bilinear current width. In direct analogy with conventional weak X-ray scattering, this fraction is determined by the effective crystal length and is less than one for frequency conversion over a long enough crystal. Figure 2 a shows that for crystal lengths less than $\sim 1 \mu \mathrm{m}$, the current SFG process accepts the full angle-energy width of the bilinear current. Figure $2 b, c$ shows that the SFG angular width is not reduced for crystal lengths up to $500 \mu \mathrm{m}$ but that the energy width is reduced, to $\sim 140 \mathrm{meV}$ at $500 \mu \mathrm{m}$.
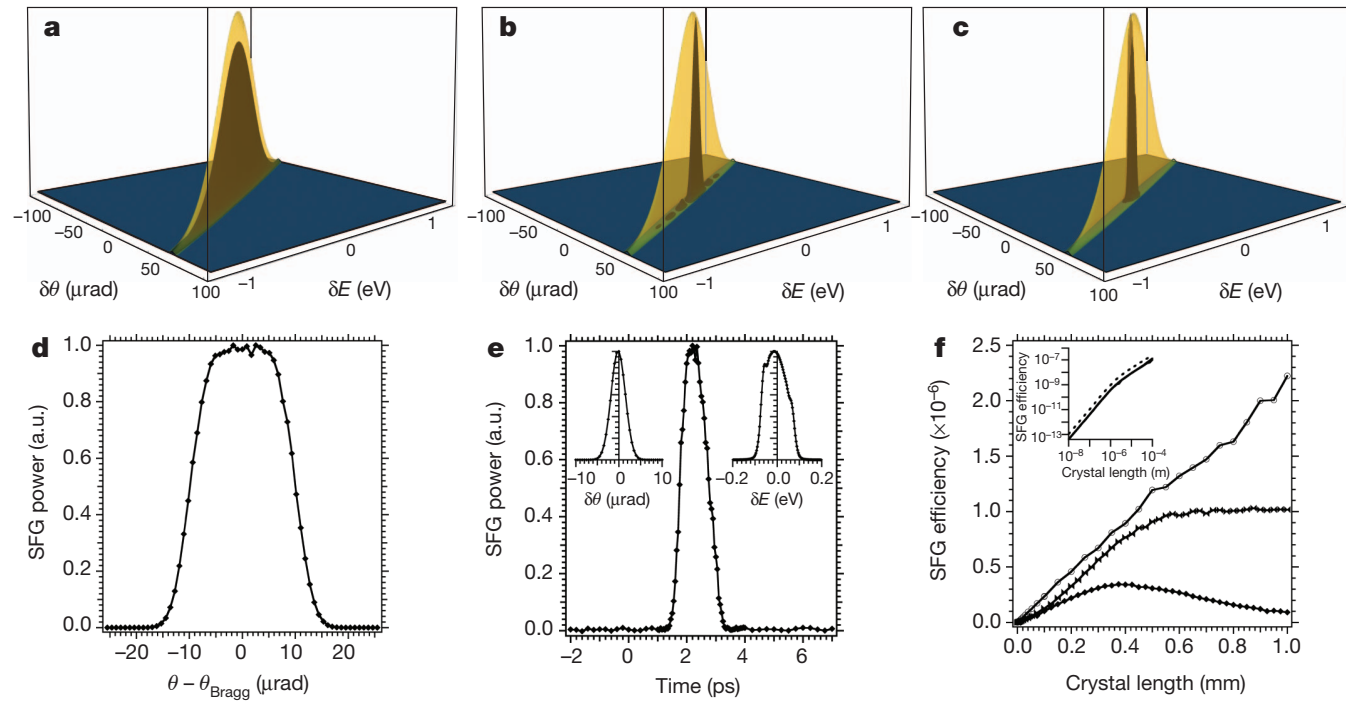

Figure 2 Wave equation simulations. All quoted widths are full-widths at half-maximum. a-c, SFG power distribution (brown) in angle-energy space for sample thicknesses of $1 \mu \mathrm{m}$ (a), $10 \mu \mathrm{m}$ (b) and $500 \mu \mathrm{m}$ (our sample; c). Deviations $\delta \theta$ and $\delta E$ from the central SFG angle and energy are plotted. The respective widths are $3.7 \mu \mathrm{rad}$ and $960 \mathrm{meV}$ (a), $3.7 \mu \mathrm{rad}$ and $210 \mathrm{meV}(\mathbf{b})$, and $3.6 \mu \mathrm{rad}$ and $140 \mathrm{meV}$ (c). The driving bilinear current is also shown (3.7 $\mu \mathrm{rad}$ and $1 \mathrm{eV}$; gold). d, SFG power versus deviation from the optimum sample angle,

for a $500-\mu \mathrm{m}$ crystal. The rocking-curve width is $20 \mu \mathrm{rad}$. e, SFG pulse duration $(\sim 1 \mathrm{ps})$, angle spread $(\sim 4 \mu \mathrm{rad}$, left inset) and energy spread $(\sim 140 \mathrm{meV}$, right inset), for a $500-\mu \mathrm{m}$ crystal. f, SFG power versus crystal length with X-ray absorption (diamonds), without absorption for a 1-ps optical pulse (bowties) and without absorption for a long (200-ps) optical pulse (circles). Inset, logscale plot. The dashed line represents the data for the long optical pulse. The other two cases (solid lines) are indistinguishable. 
The calculated SFG rocking-curve width, temporal width and efficiency variation with crystal length are shown in Figs $2 \mathrm{~d}-\mathrm{f}$. The rocking-curve width ( $20 \mu \mathrm{rad}$; Fig. $2 \mathrm{~d})$ is consistent with the experimentally determined lower limit $(\sim 8 \mu \mathrm{rad})$ and, as with conventional weak X-ray diffraction, is determined by both the effective crystal length and the angle-energy spread of the input beams. The SFG temporal width $(\sim 1$ ps; Fig. $2 \mathrm{e})$ is larger than the product $(\sim 80 \mathrm{fs})$ of perfectly overlapped X-ray ( $\sim 80 \mathrm{fs})$ and optical $(\sim 2 \mathrm{ps})$ pulses because these two pulses move through the crystal at different speeds and in different directions. With regard to dependence on crystal length, the SFG intensity decreases for crystals longer than $\sim 400 \mu \mathrm{m}$ owing to X-ray absorption (Fig. 2f). In the absence of absorption, the X-ray/optical velocity mismatch combined with our non-collinear geometry (different beam directions) leads to saturation of SFG efficiency as a function of crystal length (Fig. 2f) because the pulses eventually separate from one another. Without the velocity mismatch, the increase of efficiency with crystal length no longer saturates (Fig. 2f). The efficiency also improves with increased input-beam collimation, monochromaticity or both, to $\sim 10^{-3}$ for X-ray energy and angle widths of $0.1 \mathrm{meV}$ and $1 \mu \mathrm{rad}$ and optical energy and angle widths of $10 \mathrm{meV}$ and $1 \mathrm{mrad}$, respectively. This assumes a $500-\mu \mathrm{m}$-long crystal and neglects loss due to absorption.

Equations (1) and (2) show that the fundamental unknown specifying $E_{\mathrm{s}} / E_{\mathrm{x}}$ and, therefore, the SFG efficiency is the optically induced charge $\delta \rho_{\mathrm{o}}(Q)$; it is determined by requiring that the wave equation model reproduce the measured SFG efficiency $\left(3 \times 10^{-7}\right)$, a requirement satisfied by $\delta \rho_{\mathrm{o}}(Q) \approx 0.84 \times 10^{-4}$ electrons per cubic ångström $\left(e^{-} \AA^{-3}\right)$. Furthermore, we define a nonlinear susceptibility $\left(\chi_{\mathrm{NL}}\right)$ such that $J_{\mathrm{NL}}$ is written as a product of $E_{\mathrm{X}}, E_{\text {light }}$ and $\chi_{\mathrm{NL}}$ rather than in terms of $\delta \rho_{\mathrm{o}}(Q)$. The measured efficiency corresponds to a nonlinear susceptibility of $\chi_{\mathrm{NL}}\left(\omega_{\mathrm{o}}+\omega_{\mathrm{x}}, G_{111}\right) \approx 1.6 \times 10^{-14}$ e.s.u. Finally, the measured quantity $\delta \rho_{\mathrm{o}}(Q)$ determines the microscopic field $\delta E_{\mathrm{o}}(Q)$ associated with this charge because the two are related by Gauss's law ${ }^{3-6}$. Here $\delta E_{\mathrm{o}}(Q)$ is the $Q$ th Fourier component of the microscopic field arising in diamond as a result of the application of the external field $E_{\text {light }}\left(\right.$ refs 3-6). We find that $\left|\delta E_{\mathrm{o}}\left(Q=k_{\mathrm{o}}+G_{111}\right)\right| \approx 0.47$ in units of the macroscopic internal optical field and $\sim 0.18$ in units of the unscreened external optical field. The uncertainty in $\delta \rho_{\mathrm{o}}(Q), \chi_{\mathrm{NL}}\left(G_{111}\right)$ and $\delta E_{\mathrm{o}}(Q)$ is $\sim 40 \%$, determined by the estimated uncertainty of a factor of two in the measured efficiency. The connections between the efficiency, induced charge, nonlinear susceptibility and induced microscopic field are discussed in Supplementary Information, section 2.

\section{Optically polarized valence charge}

Associating optically polarized charge with the charge components of interest in a material is important for X-ray/optical SFG pump-probe applications. Here we consider atomic-scale optical polarization in diamond. Models that assign all optical polarizability to covalent bond charge, here termed bond charge models, have had success describing the macroscopic optical response of covalent semiconductors ${ }^{30,31}$. To test whether this idea can be extended to describe atomic-scale polarizability, we compare measured charge densities with those calculated from first principles. We find that the Fourier-space induced charge computed using density functional theory ${ }^{32,33}\left(\delta \rho_{\mathrm{o}}\left(G_{111}\right)=1.07 \times 10^{-4} e^{-} \AA^{-3}\right.$; Methods; see also http://www.quantum-espresso.org) is, to within the $\sim 40 \%$ experimental error, consistent with the measured charge density $\left(\sim 0.84 \times 10^{-4} e^{-} \AA^{-3}\right)$.

A real-space picture of the induced charge is required to assess whether light primarily polarizes bond charge. Such a real-space reconstruction is beyond the scope of the experiments reported here. Instead, we compute the real-space induced charge distribution using density functional theory. The computed ground-state valence charge density (Fig. 3a, d) shows that the covalent bond is characterized by two charge accumulation peaks, one on each side of the bond midpoint, a feature confirmed by X-ray scattering data ${ }^{28}$. The corresponding induced charge calculation (Fig. 3b, e) shows that optical polarization is predominantly expressed at the charge accumulation peaks of the covalent bond; this can be seen by comparing the positions of peak bond charge density and peak optically induced charge density (Fig. 3c, f). We find that the induced charge varies linearly with optical field strength and, for the optical field strength of the current experiment, corresponds to a $0.2 \%$ modification to the charge density at the charge accumulation peak. The density functional theory calculations therefore support a view that light predominantly perturbs chemical bonds in the lattice. We note that EUV radiation $(90 \pm 30 \mathrm{eV})$ predominantly polarizes charge at the bond midpoint rather than at the charge accumulation peaks ${ }^{23}$.

Finally, we have constructed a simple semi-empirical bond charge model in the spirit of ref. 1 (Supplementary Information, section 1) and find that to within our estimated measurement uncertainty, a multiplicative factor of $\sim 1.4$, the bond charge model predicts a charge density $\left(\sim 1.3 \times 10^{-4} e^{-} \AA^{-3}\right)$ in reasonably good agreement with the measured charge density $\left(\sim 0.84 \times 10^{-4} e^{-} \AA^{-3}\right)$. The bond charge model uses measured data: the macroscopic optical response and the microscopic bond charge distribution. An assessment of this model is therefore primarily an assessment of the assumption that optical polarizability resides in the bond charge. Although confirmation in the form of an experimental real-space reconstruction of the induced charge is required, reasonable agreement between measured densities and those predicted by the bond charge model lends further support to the identification of bond charge as the optically polarizable charge.

\section{Probing dynamics with $\mathrm{X}$-ray wave mixing}

Ultrafast X-ray diffraction (UXD) is a well-established technique that has made important contributions to advancing our understanding of molecular and material dynamics ${ }^{34-38}$. The method uses time-delayed $\mathrm{X}$-ray pulses to follow ionic cores after sudden excitation, often by an optical pulse. Ionic cores are followed because compact charge about the nucleus, or 'core' charge, typically dominates the electron density in a material. Importantly, information on the chemically relevant valence charge is typically not obtained in experiments using ultrafast $\mathrm{X}$-ray diffraction. X-ray wave mixing provides a way to probe the valence charge density in a material. It can be used to probe both the initial perturbation of a light-driven process as well as the subsequent valence charge dynamics. We first consider the initial perturbation.

The perturbation initiating a light-driven process is often poorly understood and, so far, has not been directly measurable. An X-ray pulse can accompany a perturbing optical pulse to probe the initial valence charge distortion directly by means of X-ray/optical SFG. A real-space reconstruction of the induced charge can, in principle, be obtained by measuring multiple Fourier components and using crystallographic phasing methods ${ }^{39,40}$. As with conventional crystallography, the achievable spatial resolution is, through Bragg's law, set by the scattering angle ${ }^{40}$. A less challenging method is to measure a small set of SFG Fourier amplitudes to distinguish between competing model descriptions of the initial perturbation. For instance, magneto-optical Kerr measurements (S. Roy et al., manuscript in preparation) suggest that optical radiation perturbs the helical spin structure in antiferromagnetic dysprosium thin films. However, the underlying mechanism is unclear: light might directly couple to localized spin-aligned $f$ electrons or the direct coupling may instead be to delocalized metallic electrons. By measuring a small number of X-ray/ optical SFG Fourier amplitudes, it should be possible to determine the degree to which the initial optical excitation is localized and therefore to distinguish between these possibilities.

Beyond probing initial optical perturbations, the evolution of valence charge in a material can be probed with a time-delayed pulse pair: an X-ray/optical pair to probe optically responsive charge or an $\mathrm{X}$-ray/EUV pair to probe a significantly larger fraction of the valence charge $e^{8,20,23}$. Unfavourable frequency scaling of the electronic polarizability suggests that the efficiency of X-ray/EUV SFG is significantly 

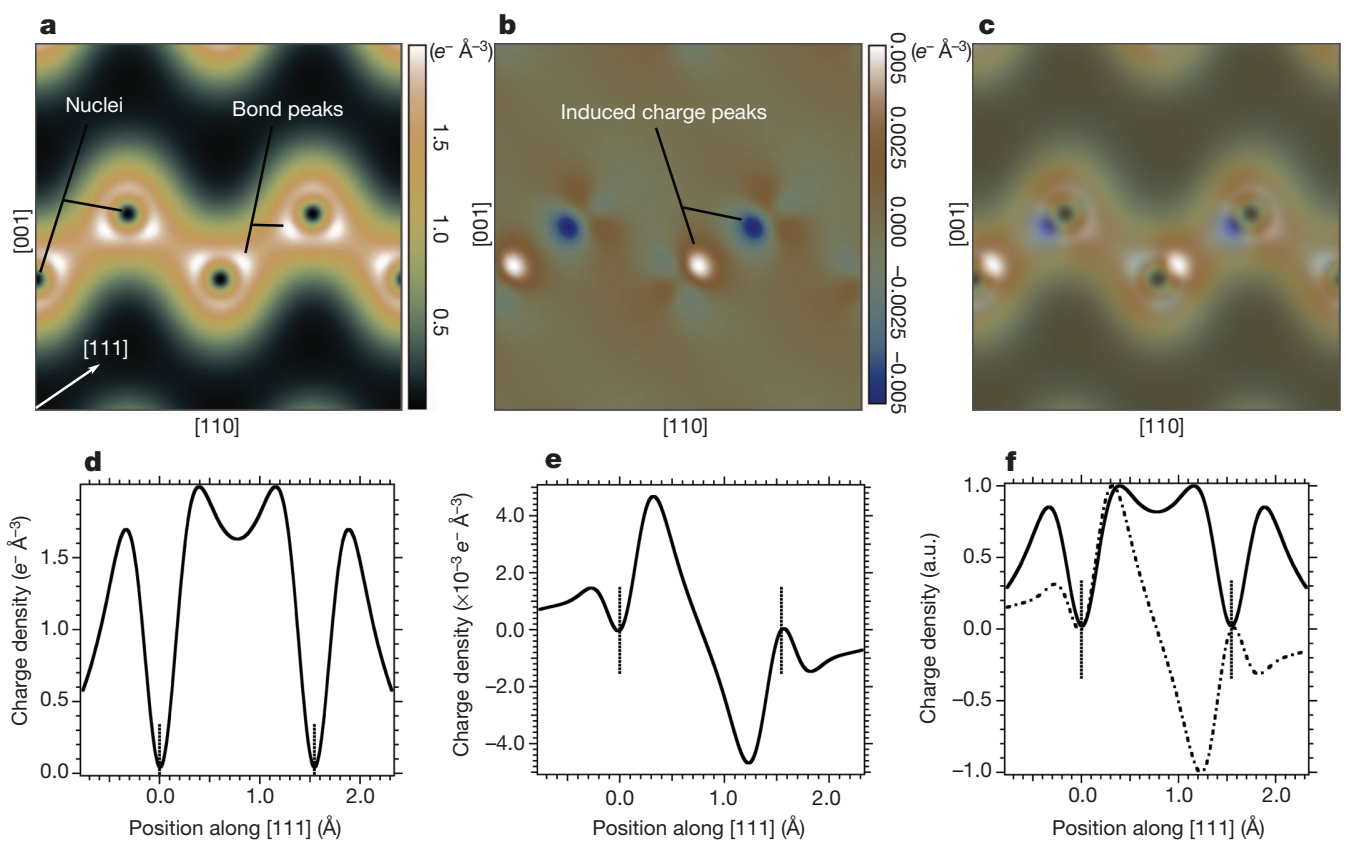

Figure $3 \mid$ Density functional theory calculations of real-space valence charge density in diamond. a, Ground-state charge density (colour scale) showing the covalent bond charge accumulation peaks. b. Induced charge density produced by a constant field $\left(2.7 \times 10^{8} \mathrm{~V} \mathrm{~m}^{-1}\right)$ in the [111] direction. c, Induced charge image (b) overlaid on the unperturbed charge image (a), demonstrating that optical polarization is primarily found at the charge

lower than that of X-ray/optical SFG. We calculate that the bilinear current (the square root of the efficiency) for X-ray $(8,000 \mathrm{eV}) / \mathrm{EUV}$ $(100 \mathrm{eV}) \mathrm{SFG}$ is $\sim 5,000$ times smaller than that for X-ray $(8,000 \mathrm{eV}) /$ optical (1.55 eV) SFG (Supplementary Information, section 4). Although X-ray/EUV SFG could in principle be used to construct time-dependent valence charge density maps, the expected low efficiency may make this an application to which future light sources are better suited. For experiments in which the significantly lower signal rate is not acceptable, X-ray/optical SFG can be used to probe a selected portion of the valence charge.

A material's optical polarizability is not uniformly distributed across the valence charge and, rather than probing the full valence charge distribution, X-ray/optical SFG selectively probes the optically polarizable component. To the extent that the optically polarizable charge can, through measurement and theory, be correctly associated with charge components of interest in a material, it should be possible to obtain novel dynamical information. For instance, assuming, as suggested above, that the optically polarizable charge in diamond is bond charge, chemical bond dynamics can be studied by measuring the time evolution of select X-ray/optical SFG Bragg peaks. Ultrafast $\mathrm{X}$-ray diffraction experiments using the temporal behaviour of conventional Bragg peaks have offered insight into, among other things, charge ordering dynamics in complex materials ${ }^{35}$, coherent atomic motion associated with both phonon ${ }^{36}$ and phonon-polariton ${ }^{37}$ propagation, and bond softening in optically excited materials ${ }^{38}$. Analogous experiments that probe valence charge should provide additional insight. For instance, the coupling between ionic cores and chemical bonds could be studied: induced coherent ionic-core oscillations will induce chemical bond oscillations that may differ from the core oscillations in any combination of phase, amplitude and frequency. Similarly, bond delocalization or breaking would have a clear signature in the evolution of selected X-ray/optical SFG Bragg peaks.

Finally, to comment on the viability of X-ray/optical SFG at existing synchrotrons, we estimate that if the same experimental apparatus (including a $1-\mathrm{kHz}$ optical laser) were used in conjunction with a accumulation peaks. $\mathbf{d}-\mathbf{f}$, Charge density along the [111] direction and through the bond peaks for $\mathbf{a}-\mathbf{c}$, respectively. The bond peaks $(0.39$ and $1.16 \AA$; $)$ and induced charge peaks ( 0.32 and $1.23 \AA$; e) are evident; $\mathbf{f}$ shows normalized densities for both the ground-state (solid) and the induced (dashed) charge distributions.

third-generation synchrotron such as the Advanced Photon Source, it would take $\sim 4,000$ times longer to obtain data with the same statistics (Supplementary Information, section 6). By using highrepetition-rate $(1-\mathrm{MHz})$ optical lasers, it may be possible to approach the count rate of the current experiment at a third-generation synchrotron (Supplementary Information, section 6).

\section{Conclusion}

We have demonstrated X-ray/optical SFG, a new tool for probing light-matter interactions on the atomic scale. To within experimental errors of $\sim 40 \%$, the measured conversion efficiency $\left(3 \times 10^{-7}\right)$ is consistent with first-principles calculations of atomic-scale optical polarization in diamond. This measurement, in combination with $a b$ initio simulations, supports the extension to atomic length scales of the long-held supposition that light selectively polarizes chemical bonds in covalent semiconductors. This conclusion is tentative and must be confirmed by an experimental real-space reconstruction of the optically polarized charge.

More generally, X-ray wave mixing creates new opportunities for probing matter. X-ray/optical SFG provides access to two closely related and previously inaccessible quantities: the induced charges and the associated microscopic fields that arise when light illuminates a material. The ability to measure optically induced microscopic fields provides novel data for benchmarking condensed-matter theories. Similarly, X-ray/optical SFG and the closely related X-ray/EUV SFG process create new opportunities to study dynamics by directly probing valence charge on atomic time and length scales.

\section{METHODS SUMMARY}

Experiment. Experiments were performed at the Linac Coherent Light Source ${ }^{26}$ (LCLS). The 8 -keV LCLS beam $(\sim 1.7 \mu \mathrm{rad}, \sim 20$-eV full-width at half-maximum, $\sim 80 \mathrm{fs})$ passed through a $\mathrm{Si}(111)$ monochromator and was directed to a $(100)$ surface-cut diamond sample oriented for Laue geometry diffraction from the (111) planes. X-ray/optical SFG was observed using $800-\mathrm{nm}$ optical pulses $\left(\sim 1.5 \times 10^{10} \mathrm{~W} \mathrm{~cm}^{-2}\right)$ stretched to a duration $(\sim 2 \mathrm{ps})$ long compared with the X-ray/optical timing jitter ( $\sim 200 \mathrm{fs}$ ). Switching from elastic diffraction to SFG was accomplished by rotating the diamond sample by $\sim 70 \mu \mathrm{rad}$, moving the 
analyser crystal to the expected SFG beam position and then rotating the analyser to increase the central pass energy by $1.55 \mathrm{eV}$ ( $h v_{\text {optical }}$, where $v_{\text {optical }}$ is the optical frequency). Scattered X-rays were wavelength-resolved with a Si(220) channelcut crystal.

Variability in wave equation predictions. Input beams are modelled as linearly chirped Gaussians with spatial and temporal chirp parameters adjusted to match angle and energy widths: $\sim 4.1 \pm 1 \mathrm{mrad}$ and $68 \pm 10 \mathrm{meV}$ for the optical beam and $\sim 1.7 \pm 1 \mu \mathrm{rad}$ and $1 \pm 0.1 \mathrm{eV}$ for the $\mathrm{X}$-ray beam. The SFG efficiency varies by less than $2 \%$ for the stated variations in all parameters except for X-ray energy. We estimate an uncertainty of $10 \%$ in the X-ray bandwidth $(1 \pm 0.1 \mathrm{eV})$ and determine that this corresponds to an uncertainty of $10 \%$ in the predicted SFG efficiency, which is small compared with the uncertainty (of a factor of two) in the measured SFG efficiency. We considered two X-ray pulse models: an $\sim 80$-fs (actual duration) pulse with a $1-\mathrm{eV}$ bandwidth accounted for by a linear frequency chirp, and a transform-limited $(\sim 2$-fs $)$ pulse with a 1 -eV bandwidth We find that the SFG efficiency and pulse duration are insensitive to these choices, varying by less than $2 \%$.

Full Methods and any associated references are available in the online version of the paper.

\section{Received 14 February; accepted 18 June 2012}

1. Freund. I \& Levine, B. F. Optically modulated X-ray diffraction. Phys. Rev. Lett. 25, $1241-1245(1970)$

2. Eisenberger,P. M. \& McCall, S. L. Mixing of X-ray and optical photons. Phys. Rev. A 3, 1145-1151 (1971)

3. Jackson, J. D. Classical Electrodynamics (Wiley, 1975)

4. Arya, K. \& Jha, S. S. Microscopic optical fields and mixing coefficients of $x$-ray and optical frequencies in solids. Pramana 2, 116-125 (1974).

5. Adler, S. L. Quantum theory of the dielectric constant in real solids. Phys. Rev. $\mathbf{1 2 6}$ 413-420 (1962)

6. Pine, A. S. Self-consistent-field theory of linear and nonlinear crystalline dielectrics including local-field effects. Phys. Rev. 139, A901-A911 (1965).

7. Woo, J. W. F. \& Jha, S. S. Inelastic scattering of $x$ rays from optically induced chargedensity oscillations. Phys. Rev. B 6, 4081-4082 (1972).

8. Freund, I. Nonlinear X-ray diffraction: determination of valence electron charge distributions. Chem. Phys. Lett. 12, 583-588 (1972).

9. Van Vechten, J. A., \&. Martin, R. M. Calculation of local effective fields: optical spectrum of diamond. Phys. Rev. Lett. 28, 446-449 (1972)

10. Freund, I\&. Levine, B. F. Surface effects in the nonlinear interaction of X-ray and optical fields. Phys. Rev. B 6, 3059-3060 (1973).

11. Arya, K. \& Jha, S. S. Microscopic optical fields in diamond and germanium: molecular-orbital approach. Phys. Rev. B 10, 4485-4487 (1974).

12. Johnson, D. L. Local field effects and the dielectric response matrix of insulators: $A$ model. Phys. Rev. B 9, 4475-4484 (1974).

13. Schweigert, I. V. \& Mukamel, S. Coherent ultrafast core-hole correlation spectroscopy: X-ray analogues of multidimensional NMR. Phys. Rev. Lett. 99, 163001 (2007)

14. Tanaka, S. \& Mukamel, S. Probing exciton dynamics using Raman resonances in femtosecond X-ray four-wave mixing. Phys. Rev. A 67, 033818 (2003).

15. Nazarkin, A., Podorov, S., Uschmann, I., Forster, E. \& Sauerbrey, R. Nonlinear optics in the angstrom regime: hard-X-ray frequency doubling in perfect crystals. Phys. Rev. A 67, 041804(R) (2003).

16. Tanaka, S. \& Mukamel, S. Coherent X-ray Raman spectroscopy: a nonlinear local probe for electronic excitations. Phys. Rev. Lett. 89, 043001 (2002).

17. Hudis, E., Shkolnikov, P. L. \& Kaplan, A. E. X-ray stimulated Raman scattering in Li and He. Appl. Phys. Lett. 64, 818-820 (1994).

18. Freund, I. \& Levine, B. F. Parametric conversion of $X$ rays. Phys. Rev. Lett. 23, 854-857 (1969).

19. Eisenberger, P. \& McCall, S. L. X-ray parametric conversion. Phys. Rev. Lett. 26 , 684-688 (1971)

20. Danino, H. \& Freund, I. Parametric down conversion of $X$ rays into the extreme ultraviolet. Phys. Rev. Lett. 46, 1127-1130 (1981).

21. Yoda, Y., Suzuki, T., Zhang, X. W., Hirano, K. \& Kikuta, S. X-ray parametric scattering by a diamond crystal. J. Synchrotron Radiat. 5, 980-982 (1998).
22. Adams, B. et al. Parametric down conversion of X-ray photons. J. Synchrotron Radiat. 7, 81-88 (2000)

23. Tamasaku, K. Sawada, K. Nishibori, E. \& Ishikawa, T. Visualizing the local optical response to extreme-ultraviolet radiation with a resolution of $\lambda / 380$. Nature Phys. 7, 705-708 (2011)

24. Platzman, P. M. \& Isaacs, E. D. Resonant inelastic X-ray scattering. Phys. Rev. B 57, $11107-11114$ (1998)

25. Kotani, A. \& Shin, S. Resonant inelastic X-ray scattering spectra for electrons in solids. Rev. Mod. Phys. 73, 203-246 (2001).

26. Emma, P. et al. First lasing and operation of an ångstrom-wavelength free-electron laser. Nature Photon. 4, 641-647 (2010).

27. Palik, E. D. (ed.) Handbook of Optical Constants of Solids 313-334 (Academic, 1998).

28. Spackman, M. A. The electron distribution in diamond: a comparison between experiment and theory. Acta Crystallogr. A 47, 420-427 (1991).

29. Shen, Y. R. The Principles of Nonlinear Optics 5-40 (Wiley, 1984).

30. Phillips, J. C. Covalent bond in crystals. I. Elements of a structural theory. Phys. Rev. $166,832-838$ (1968)

31. Levine, B. F. Electrodynamic bond-charge calculation of nonlinear optical susceptibilities. Phys. Rev. Lett. 22, 787-790 (1969).

32. Giannozzi, P. et al. Quantum Espresso: a modular and open-source software project for quantum simulations of materials. J. Phys. Condens. Matter 21, 395502 (2009).

33. Souza, I. Iniguez, J. \& Vanderbilt, D. First principles approach to insulators in finite electric fields. Phys. Rev. Lett. 89, 117602 (2002)

34. Ihee, H. etal. Ultrafast X-ray diffraction of transient molecular structures in solution Science 309, 1223-1227 (2005).

35. Möhr-Vorobeva, E. et al. Nonthermal melting of a charge density wave in $\mathrm{TiSe}_{2}$ Phys. Rev. Lett. 107, 036403 (2011)

36. Lindenberg, A. M. et al. Time-resolved X-ray diffraction from coherent phonons during a laser-induced phase transition. Phys. Rev. Lett. 84, 111-114 (2000).

37. Cavalleri, A. et al. Tracking the motion of charges in a terahertz light field by femtosecond X-ray diffraction. Nature 442, 664-666 (2006).

38. Fritz, D. M. etal. Ultrafast bond softening in bismuth: mapping a solid's interatomic potential with X-rays. Science 315, 633-636 (2007)

39. Wu, J. S., Spence, J. C. H., O'Keeffe, M. \& Groy, T. L. Application of a modified Oszlanyi and Suto ab initio charge-flipping algorithm to experimental data. Acta Crystallogr. A 60, 326-330 (2004).

40. Coppens, P. X-Ray Charge Densities and Chemical Bonding (Oxford Univ. Press, 1997).

Supplementary Information is available in the online version of the paper.

Acknowledgements We thank J. D. Jackson, D. Vanderbilt, and J. C. H. Spence for commenting on various aspects of this work. T.E.G. was supported by the Office of Science, Office of Basic Energy Sciences, of the US Department of Energy under contract no. DE-AC02-05CH11231. The work of S.E.H. and S.S. was supported by the US Air Force Office of Scientific Research and the US Army Research Office. D.A.R. and S.G. were supported as part of the AMOS programme within the Chemical Sciences, Geosciences, and Biosciences Division, Office of Basic Energy Sciences, US Department of Energy. Portions of this research were carried out at the LCLS at SLAC National Accelerator Laboratory. LCLS is an Office of Science User Facility operated for the US Department of Energy Office of Science by Stanford University. Preliminary experiments were performed at the Advanced Light Source at Lawrence Berkeley National Laboratory.

Author Contributions T.E.G. and J.B.H. had the idea for the x-ray/optical SFG project. D.M.F., T.E.G., J.B.H., M.C.., T.K.A., J.M.F. and D.A.R. contributed to the experiment design. D.M.F., M.C., H.L., D.Z. and R.N.C. were responsible for the X-ray pump-probe instrument and the optical laser. D.M.F., M.C., T.E.G., J.B.H., H.L., D.Z., T.K.A., J.M.F., Y.F., R.N.C. D.A.R. S.S., M.F, S.G. and J.C. collected data. S.E.H., S.S., T.E.G. and T.K.A. contributed to the wave equation calculations. Data analysis was done by T.E.G., H.L. and J.M.F. Construction of the bond charge model was done by T.E.G. Density functional theory calculations were done by S.C. T.E.G. wrote the manuscript. All authors contributed to the work presented here and to the final paper.

Author Information Reprints and permissions information is available at www.nature.com/reprints. The authors declare no competing financial interests. Readers are welcome to comment on the online version of the paper. Correspondence and requests for materials should be addressed to T.E.G. (teglover@lbl.gov). 


\section{METHODS}

Experiment. Experiments were performed at the $\mathrm{LCLS}^{26}$, an X-ray free-electron laser that provided an $8-\mathrm{keV}, 60-\mathrm{Hz}$ beam with angle and energy spreads of respectively $\sim 1.7 \mu \mathrm{rad}$ and $\sim 20 \mathrm{eV}$ (full-widths at half-maximum). The $\sim 80$-fs pulse (full-width at half-maximum) contained $\sim 5 \times 10^{10}$ photons. Complications from spectral jitter were mitigated by converting spectral fluctuations to intensity fluctuations: X-rays were passed through a two-bounce $\mathrm{Si}(111)$ monochromator $(\sim 1-\mathrm{eV}$ bandpass) and a non-destructive Compton scattering diagnostic recorded the shot-by-shot beam intensity to normalize intensity fluctuations. Monochromatic X-rays $(\sim 1.7 \mu \mathrm{rad}, 1 \mathrm{eV})$ were directed to a (100)-surface-cut diamond sample oriented for 8-keV, (111) Laue geometry diffraction. A Si(220) channel-cut crystal was used to energy-resolve detected X-rays. X-ray/optical SFG was observed using $800-\mathrm{nm}$ optical pulses $\left(1.5 \times 10^{10} \mathrm{~W} \mathrm{~cm}^{-2}\right)$ stretched to a duration $(\sim 2 \mathrm{ps})$ long compared with the X-ray/optical timing jitter $(\sim 200 \mathrm{fs})$. $\mathrm{X}$-ray/optical overlap was achieved by maximizing the reduction in X-ray diffraction from an optically perturbed Bi sample.

Switching from elastic diffraction to SFG was accomplished by first rotating the diamond sample by $\delta \phi_{111} \approx 70 \mu \mathrm{rad}$ (less grazing). A two-theta arm then rotated the analyser to the expected SFG beam position, $\sim 370 \mu$ rad relative to the elastically scattered beam position before the diamond rotation. Finally, the central pass energy of the analyser was increased (such that the optical photon energy was $h v_{\text {optical }}=1.55 \mathrm{eV}$ ) by rotating the crystal itself $\left(\delta \phi_{220} \approx 85 \mu \mathrm{rad}\right)$. The sample and analyser detunings, each $\sim 10$ rocking-curve half-widths, reduced the intensity of elastically scattered (background) light reaching the detector by seven to eight orders of magnitude relative to optimized elastic scattering conditions $\left(\delta \phi_{111}=\delta \phi_{220}=0\right)$. We did not make use of the $\sim 500-\mu \mathrm{rad}$ angular separation between the elastic and SFG beams.

The X-ray flux reaching our detector was $\sim 3 \times 10^{7}$ photons per pulse under optimized elastic diffraction conditions, which is less than the initial $\sim 5 \times 10^{10}$ photons per pulse due to loss associated with both absorption and five crystal reflections (two $\mathrm{Si}(111)$, the diamond sample and two $\mathrm{Si}(220)$ ). The SFG flux reaching our detector was $\sim 65$ photons per pulse.

Variability in wave equation predictions. Input beams were modelled as linearly chirped Gaussians with spatial and temporal chirp parameters adjusted to match the corresponding angle and energy widths: The optical beam had an energy spread of $68 \mathrm{meV}$ and an in-vacuum angle spread of $\sim 4.1 \mathrm{mrad}$. A linear frequency chirp stretched the optical pulse to a duration $(\sim 2 \mathrm{ps})$ long compared with the X-ray/optical timing jitter ( $\sim 200 \mathrm{fs})$. The X-ray angle spread $(\sim 1.7 \mu \mathrm{rad})$ was set by the LCLS divergence whereas the energy spread $(\sim 1 \mathrm{eV})$ was set by the $\mathrm{Si}(111)$ monochromator. Two X-ray pulse models were considered: one with a pulse duration equal to the actual LCLS duration $(\sim 80 \mathrm{fs})$ and a $1-\mathrm{eV}$ bandwidth accounted for by a linear frequency chirp, and one with a transformlimited pulse with a duration $(\sim 2 \mathrm{fs})$ determined by the $1-\mathrm{eV}$ bandwidth. We find that the SFG efficiency and pulse duration are insensitive to these choices; each varies by less than $2 \%$. Insensitivity of efficiency to the specific duration of the (short) X-ray pulse might be expected because for either pulse duration the $\mathrm{X}$-ray pulse is short compared with the optical pulse and therefore sees the same optical field strength. Similarly, pulse duration insensitivity seems reasonable because the $\mathrm{X}$-ray and optical pulses move through the crystal at different speeds; this causes the relative $\mathrm{X}$-ray/optical time delay to vary linearly across the crystal. The magnitude of this 'time shift' ( $>1 \mathrm{ps})$ is large compared with either X-ray pulse duration considered ( 80 or $2 \mathrm{fs}$ ). The actual LCLS pulse is probably a random distribution of transform-limited spikes within a $\sim 80$-fs temporal envelope.

Finally, the predicted efficiency depends on the degree to which the input beams are collimated and/or monochromatic; here we consider how much the predicted efficiency varies owing to uncertainty in the angle-energy spread of the input beams. We estimate the uncertainties in the X-ray energy, laser wavelength, X-ray angle and laser angle 'widths' to be $\pm 0.1 \mathrm{eV}, \pm 10 \mathrm{meV}$, $\pm 1 \mu \mathrm{rad}$ and $\pm 1 \mathrm{mrad}$, respectively. The variation in the predicted SFG efficiency is less than $2 \%$ for the stated variations in all parameters except for X-ray energy. The variation in efficiency with X-ray energy spread is approximately linear. The bandwidth of the $\mathrm{X}$-ray beam should be known to good precision because it is set by the $\mathrm{Si}(111)$ monochromator acceptance. We estimate an uncertainty of $\sim 10 \%$ in the X-ray bandwidth $(1 \pm 0.1 \mathrm{eV})$, which corresponds to a variation of $\sim 10 \%$ in the predicted efficiency. This uncertainty is small compared with the uncertainty in the measured SFG efficiency, which is a factor of two as determined by variability in the measured X-ray/SFG pulse energy. 
The Supplemental Information is divided into seven sections. Section 1 (Density function theory calculations) discusses the first-principles calculations of microscopic optical polarization in diamond. Section 2 (Bond charge model) details how the bond charge model is constructed. Using empirical data, this semi-empirical and phenomenological model predicts the microscopic optical polarization in diamond. In section 3 (Relationship between SFG efficiency, induced charge, nonlinear susceptibility, and microscopic field) we derive the connection between the quantities listed in this section title. In section 4 (Derivation of Wave Equation) we derive the specific form of the wave equation used to model x/o SFG. In section 5 (Relative nonlinear current amplitudes) we compute the relative amplitudes of the various nonlinear current terms contributing to SFG. In section 6 (Derivation of nonlinear current terms) we derive the equations used to compute the nonlinear current amplitudes. Finally, in section 7 (Xray/optical SFG at other light sources) we compare relative SFG signal levels at various $\mathrm{x}$-ray sources. 


\section{Supplemental Information}

\section{Density functional theory calculations}

To calculate the induced charge density in diamond, we use density functional theory (DFT) with the generalized gradient approximation and ultrasoft pseudopotentials as implemented in the Quantum Espresso software package ${ }^{30}$. First we fully relax the unit cell lattice parameter (obtaining $\mathrm{a}=3.567 \AA$ ) and then apply a constant external electric field using the Berry phase method ${ }^{31}$. The field strength/direction are equal to that of the experimental optical field. The field strength (vacuum field amplitude less loss due to surface reflectivity) is $\mathrm{E}_{\text {light }}=3.4 \times 10^{8} \mathrm{~V} / \mathrm{m}$ and the component along [111] is $E_{\text {light }}=2.7 \times 10^{8} \mathrm{~V} / \mathrm{m}$. By comparing the Fourier component of charge density with and without the electric field we calculate the charge density difference (i.e. induced charge). The induced charge density $\rho\left(G_{111}, E=3.4 \times 10^{8}\right)-\rho\left(G_{111}, E=0\right)$ equals $1.07 \times 10^{-4}$ $\mathrm{e} / \AA^{3}$. By varying the electric field strength we determine that the induced charge varies linearly with field strength. At the location where the induced charge is largest and for the current field strength, the induced charge amounts to a $0.2 \%$ change to the unperturbed charge density. Further, the induced charge density component, $\rho\left(G_{111}, E\right)$, is due entirely to the [111] component of $\mathrm{E}_{\text {light }}$; the orthogonal component has negligible effect. We confirmed that using the local density approximation instead of the generalized gradient approximation changes the induced charge density by only $1 \%$ and that changing the type of pseudopotential from ultrasoft to normconserving modifies the induced charge density by only $2 \%$.

A view of the DFT-computed valence charge distribution in real-space is shown in Fig. SI1. 


\section{Supplemental Information}



Figure SI1. Real-space view of valence charge distribution in diamond, as computed using density functional theory. The figure shows both the unperturbed and opticallyperturbed valence charge distribution in diamond. The images were rendered using a hardware-accelerated 3-D texture-based direct volume rendering of a simulated $100^{3}$ scalar data field. Intensity and gradient-based opacity and color transfer functions show valence electron density in green and the optically induced charge differences in orangered (increased charge density relative to unperturbed) and blue (decreased). Image courtesy of Dr. Joerg Meyer, Lawrence Berkeley National Laboratory (Visualization Group). 


\section{Supplemental Information}

\section{Bond charge model}

Historically, it was found that x-ray scattering data for diamond could be understood in terms of a two component charge density : an 'atomic' component exhibiting spherical symmetry about the nuclei and a 'bond' component lacking this symmetry ${ }^{41}$. The 'bond' component was identified as the polarizable charge in the lattice : Phillips argued that there was a close relationship between the covalent bond charge and the macroscopic dielectric function ${ }^{32}$, and subsequently Levine successfully described optical nonlinear susceptibilities in terms of bond charge motion ${ }^{33}$.

Though the separation of valence charge into 'atomic' and 'bond' components is not unique, this strategy has been helpful in constructing simple models of optical polarizability and we adopt this strategy to construct, in essence, the same model for microscopic optical polarizability as discussed in [1]. The bonds are assumed to be the polarizable sites in the unit cell. One predicts both the magnitude and microscopic spatial distribution of induced charge by : 1. making use of the measured macroscopic optical susceptibility $^{27}$ and 2. assuming that the spatial distribution of induced charge is identical to the measured ${ }^{28}$ spatial distribution of bond charge.

The induced charge is written as the divergence of the induced polarization :

$$
\delta \rho\left(\mathbf{r}, \mathbf{E}_{\text {light }}\right)=-\nabla \cdot \mathbf{P}\left(\mathbf{r}, \mathbf{E}_{\text {light }}\right)
$$

The total polarization is written as a sum over the polarization at individual bond sites in the unit cell :

$$
\mathbf{P}\left(\mathbf{r}, \mathbf{E}_{\text {light }}\right)=\Sigma_{\text {Bonds j }} \mathbf{p}_{\mathrm{j}}\left(\mathbf{r}, \mathbf{E}_{\text {light }}\right)
$$

Following [1], the polarization is related to the optical field $\left(\mathbf{E}_{\text {light }}\right)$ by defining a microscopic optical susceptibility (polarizability per volume) $\boldsymbol{\alpha}^{\prime}(\mathbf{r})$ : 


\section{Supplemental Information}

$$
\mathbf{p}_{j}\left(\mathbf{r}, \mathbf{E}_{\text {light }}\right) \equiv \boldsymbol{\alpha}_{\mathrm{j}}{ }^{\prime}(\mathbf{r}) \cdot \mathbf{E}_{\text {light }}
$$

Consistent with our assumption that only bonds are optically polarizable, we write the microscopic optical susceptibility as the magnitude of the average bond polarizability $\left(\alpha_{\mathrm{o}}\right)$ times the bond charge spatial distribution function $\delta \rho_{\text {bond }}(\mathbf{r})$ :

$$
\boldsymbol{\alpha}_{\mathrm{j}}{ }^{\prime}(\mathbf{r})=\alpha_{\mathrm{o}} \delta \rho_{\mathrm{j}, \text { bond }}(\mathbf{r}) \mathbf{I}
$$

Here $\mathbf{I}$ is the unit dyadic ${ }^{1}$. The bond charge distribution $\left(\delta \rho_{\text {bond }}\right)$ is normalized; its integral over space is unity. Inserting (4) into (3) leads to :

$$
\mathbf{p}_{\mathrm{j}}\left(\mathbf{r}, \mathbf{E}_{\text {light }}\right)=\alpha_{\mathrm{o}} \delta \rho_{\mathrm{j}, \text { bond }}(\mathbf{r}) \mathbf{E}_{\text {light }}
$$

Inserting (5) into (2) and expanding $\mathbf{P}\left(\mathbf{r}, \mathbf{E}_{\text {light }}\right)$ in a Fourier series leads to :

$$
\Sigma_{\mathrm{Q}^{\prime}} \mathbf{P}_{\mathrm{Q}^{\prime}} \operatorname{Exp}\left[\mathrm{i} \mathbf{Q}^{\prime} \cdot \mathbf{r}\right]=\Sigma_{\text {Bonds j }} \quad \alpha_{\mathrm{o}} \delta \rho_{\mathrm{j}, \text { bond }}(\mathbf{r}) \mathbf{E}_{\text {light }}
$$

We are interested in a specific Fourier component and therefore multiply each side of (6) by $\operatorname{Exp}[-\mathrm{i} \mathbf{Q} \cdot \mathbf{r}]$ and integrate over the unit cell :

$$
\begin{gathered}
\Sigma_{\mathrm{Q}^{\prime}} \int_{\text {cell }} \mathbf{P}_{\mathrm{Q}^{\prime}} \operatorname{Exp}\left[\mathrm{i}\left(\mathbf{Q}^{\prime}-\mathbf{Q}\right) \cdot \mathbf{r}\right] \mathrm{d}^{3} \mathrm{r}=\int_{\text {cell }} \Sigma_{\text {Bonds j }} \alpha_{\mathrm{o}} \delta \rho_{\mathrm{j}, \text { bond }}(\mathbf{r}) \mathbf{E}_{\text {light }} \operatorname{Exp}[-\mathrm{i} \mathbf{Q} \cdot \mathbf{r}] \mathrm{d}^{3} \mathrm{r} \\
\mathbf{P}_{\mathrm{Q}} \mathrm{V}_{\text {cell }}=\left(\alpha_{\mathrm{o}} \mathbf{E}_{\text {light }}\right) \Sigma_{\text {Bonds j }} \int_{\text {cell }} \delta \rho_{\mathrm{j}, \text { bond }}(\mathbf{r}) \operatorname{Exp}[-\mathrm{i} \mathbf{Q} \cdot \mathbf{r}] \mathrm{d}^{3} \mathrm{r}
\end{gathered}
$$

Here $\mathbf{Q}=\mathbf{G}+\mathbf{k}_{\mathrm{o}}$ where $\mathbf{G}$ is the relevant reciprocal lattice vector and $\mathbf{k}_{\mathrm{o}}$ the optical wave vector. Defining $\mathrm{N}_{\text {cell }}=1 / \mathrm{V}_{\text {cell }}$ we obtain :

$$
\mathbf{P}_{\mathrm{Q}}=\left(\mathrm{N}_{\text {cell }} \alpha_{\mathrm{o}} \mathbf{E}_{\text {light }}\right) \Sigma_{\text {Bonds j }} \int_{\text {cell }} \delta \rho_{\mathrm{j}, \text { bond }}(\mathbf{r}) \operatorname{Exp}[-\mathrm{i} \mathbf{Q} \cdot \mathbf{r}] \mathrm{d}^{3} \mathrm{r}
$$




\section{Supplemental Information}

Next we use the Clausius-Mossotti relationship ${ }^{3}$ to express $N_{\text {cell }} \alpha_{0}$ in terms of the measured macroscopic linear optical susceptibility :

$$
\mathrm{N}_{\text {atom }} \alpha_{\text {atom }}=\{3 /(\varepsilon+2)\} \chi_{\text {macro }}
$$

where $\varepsilon$ is the dielectric constant ( $\varepsilon=1+4 \pi \chi_{\text {macro }}$. The quantities on the left side of (10) are the atom density and atom polarizability respectively. Noting that in diamond there are 8 atoms per unit cell and 2 bonds per atoms we have :

$$
\mathrm{N}_{\text {atom }} \alpha_{\text {atom }}=8 \mathrm{~N}_{\text {cell }} 2 \alpha_{o}
$$

This leads to :

$$
\mathbf{P}_{\mathbf{Q}}=\left(\chi_{\text {macro }} / 16\right)\{3 /(\varepsilon+2)\} \mathbf{E}_{\text {light }} \Sigma_{16 \text { Bonds j }} \int_{\text {cell }} \delta \rho_{\mathrm{j}, \text { bond }}(\mathbf{r}) \operatorname{Exp}[-\mathrm{i} \mathbf{Q} \cdot \mathbf{r}] \mathrm{d}^{3} \mathrm{r}
$$

In (12) we explicitly indicate that the sum is over the 16 bonds in the unit cell. The covalent bond in diamond is characterized by two equal-amplitude charge accumulation peaks $\sim 0.7 \AA$ wide (fwhm). We model each peak as a Gaussian so that a 'bond peak' becomes the fundamental polarizable unit instead of the (full) bond and the summation in (12) becomes, in practice, a summation over 32 bond peaks (two per bond). The polarization of a bond peak $\left(\alpha_{0} / 2\right)$ therefore replaces the bond polarizability $\left(\alpha_{0}\right)$ in (5). This has the effect of replacing the $1 / 16$ in (12) with $1 / 32$ and we rewrite (12) as :

$$
\mathbf{P}_{\mathrm{Q}}=\left(\chi_{\text {macro }} / 32\right)\{3 /(\varepsilon+2)\} \mathbf{E}_{\text {light }} \Sigma_{32 \text { Bond peaks } j} \int_{\text {cell }} \delta \rho_{\mathrm{j}, \text { bond }}(\mathbf{r}) \operatorname{Exp}[-\mathrm{i} \mathbf{Q} \cdot \mathbf{r}] \mathrm{d}^{3} \mathrm{r}(13)
$$

Defining the structure factor :

$$
\mathrm{F}_{\mathbf{Q}}{ }^{\mathrm{x} / \mathrm{o}} \equiv(1 / 32) \Sigma_{32 \text { Bond peaks } \mathrm{j}} \int_{\text {cell }} \delta \rho_{\mathrm{j}, \mathrm{bond}}(\mathbf{r}) \operatorname{Exp}[-\mathrm{i} \mathbf{Q} \cdot \mathbf{r}] \mathrm{d}^{3} \mathrm{r}
$$




\section{Supplemental Information}

We obtain the final expression for the polarization :

$$
\mathbf{P}_{\mathrm{Q}}=\chi_{\text {macro }} \mathrm{F}_{\mathbf{Q}}^{\mathrm{x} / \mathrm{o}}\{3 /(\varepsilon+2)\} \mathbf{E}_{\text {light }}
$$

The relevant Fourier component of the induced charge is obtained from the polarization by expanding both sides of (1) in a Fourier series to obtain :

$$
\delta \rho_{\mathbf{Q}}\left(\mathbf{E}_{\text {light }}\right)=-\mathrm{i} \mathbf{Q} \cdot \mathbf{P}_{\mathbf{Q}}\left(\mathbf{E}_{\text {light }}\right)
$$

We therefore obtain the final expression for the induced charge :

$$
\delta \rho_{\mathbf{Q}}\left(\mathbf{E}_{\text {light }}\right)=-\mathrm{i} \mathbf{Q} \cdot \mathbf{E}_{\text {light }}\{3 /(\varepsilon+2)\} \chi_{\text {macro }} \mathrm{F}_{\mathbf{Q}}{ }^{\mathrm{x} / \mathrm{o}}
$$

The Fourier component of interest for the current experiment is $\mathbf{Q}=\mathbf{G}_{111}+\mathbf{k}_{\mathrm{o}}$.

3. Relationship between SFG efficiency, induced charge, nonlinear susceptibility, and microscopic field

\section{$\underline{3.1 \text { Nonlinear susceptibility from measured efficiency }}$}

Here we obtain an expression for the nonlinear susceptibility in terms of the measured efficiency. We start with the 'optical Doppler' expression for the sum frequency current $\left(2 \mathbf{J}_{\mathrm{a}}\right.$ for $\omega_{\mathrm{x}} \gg \omega_{\text {binding }} \sim 5 \mathrm{eV}$; see equations (77) "Relative nonlinear current amplitudes" below) :

$$
\begin{aligned}
& \mathbf{J}_{\mathrm{NL}}\left(\omega_{\mathrm{x}}+\omega_{\mathrm{o}}\right)=-\mathrm{i}(\mathrm{e} / \mathrm{m})\left\{1 / \omega_{\mathrm{x}}\right\} \rho\left(\mathrm{E}_{\text {light }}^{\mathrm{G}}\right)^{\text {induced }} \mathbf{E}_{\mathrm{x}}\left(\omega_{\mathrm{x}}\right) \\
& \mathbf{J}_{\mathrm{NL}}\left(\mathbf{k}_{\mathrm{sum}}=\mathbf{k}_{\mathrm{x}}+\mathbf{k}_{\mathrm{o}}+\mathbf{G}\right)=-\mathrm{i}(\mathrm{e} / \mathrm{m})\left\{1 / \omega_{\mathrm{x}}\right\} \rho\left(\mathbf{k}_{\mathrm{o}}+\mathbf{G}\right)^{\text {induced }} \mathbf{E}_{\mathrm{x}}\left(\mathbf{k}_{\mathrm{x}}\right)
\end{aligned}
$$

Equations (18a) and (18b) are of course equivalent but are written to emphasize energy and wave vector conservation respectively. The optical wave vector is $\mathbf{k}_{\mathrm{o}}$ and, as 


\section{Supplemental Information}

implied by (16), the $\left(\mathbf{k}_{0}+\mathbf{G}\right)^{\text {th }}$ component of the induced charge is determined by the component of the optical field along the lattice vector $\mathbf{G}$ :

$$
\mathrm{E}_{\text {light }}{ }^{\mathrm{G}} \equiv \mathbf{E}_{\text {light }} \cdot \mathbf{G} /|\mathbf{G}|
$$

We are interested in a transverse (i.e. radiation) field and therefore take the transverse component of the current :

$$
\mathrm{J}_{\mathrm{NL}}{ }^{\mathrm{T}}\left(\omega_{\mathrm{x}}+\omega_{0}\right)=-\mathrm{i}(\mathrm{e} / \mathrm{m})\left\{1 / \omega_{\mathrm{x}}\right\} \rho\left(\mathrm{E}_{\text {light }}{ }^{\mathrm{G}}\right)^{\text {induced }} \mathrm{E}_{\mathrm{x}}^{\mathrm{T}}
$$

where superscript ' $T$ ' indicates the component along the sum field polarization vector $\boldsymbol{\varepsilon}_{\text {sum }}$ :

$$
\mathrm{E}_{\mathrm{x}}^{\mathrm{T}} \equiv \boldsymbol{\varepsilon}_{\text {sum }} \cdot \mathbf{E}_{\mathrm{x}}
$$

Recalling that the current is the time derivative of the polarization ${ }^{3}$ :

$$
\mathrm{J}_{\mathrm{NL}}\left(\omega_{\text {sum }}\right)=\mathrm{dP}_{\mathrm{NL}} / \mathrm{dt}=-i \omega_{\text {sum }} \mathrm{P}_{\mathrm{NL}}
$$

From (22) and (20) we obtain :

$$
\omega_{\text {sum }} \mathrm{P}_{\mathrm{NL}}{ }^{\mathrm{T}}=(\mathrm{e} / \mathrm{m})\left\{1 / \omega_{\mathrm{x}}\right\} \rho\left(\mathrm{E}_{\text {light }}{ }^{\mathrm{G}}\right)^{\text {induced }} \mathrm{E}_{\mathrm{x}}{ }^{\mathrm{T}}
$$

We next define the nonlinear susceptibility as the proportionality constant between the nonlinear polarization of interest and the relevant input field components :

$$
\mathrm{P}_{\mathrm{NL}}{ }^{\mathrm{T}}\left(\mathbf{k}_{\text {sum }}\right) \equiv \chi_{\mathrm{NL}}(\mathbf{G}) \mathrm{E}_{\text {light }}{ }^{\mathrm{G}}\left(\mathbf{k}_{\mathrm{o}}\right) \mathrm{E}_{\mathrm{x}}^{\mathrm{T}}\left(\mathbf{k}_{\mathrm{x}}\right)
$$

The fields $\mathbf{E}_{\text {light }}$ and $\mathbf{E}_{\mathrm{x}}$ are modeled as Gaussians with amplitudes $\mathrm{E}_{\text {lighta }}$ and $\mathrm{E}_{\mathrm{xa}}$. The optical field amplitude $E_{\text {lighta }}$ is known while the nonlinear susceptibility $\left(\chi_{\mathrm{NL}}\right)$ is 


\section{Supplemental Information}

unknown. We also regard the x-ray field amplitude $\left(E_{x a}\right)$ as unknown. The product $\left(\chi_{\mathrm{NL}} \mathrm{E}_{\mathrm{xa}}\right)$ is a constant and we solve the wave equation in terms of this unknown constant. The resulting SFG field amplitude is linearly proportional to the constant $\left(\chi_{N L} E_{\mathrm{xa}}\right):$

$$
\mathrm{E}_{\mathrm{SFG}}=\chi_{\mathrm{NL}} \mathrm{E}_{\text {lighta }} \mathrm{E}_{\mathrm{xa}} \mathrm{H}_{\mathrm{wave} \mathrm{eqn}}
$$

Where $\mathrm{H}_{\text {wave eqn }}$ is a function determined by solving the wave equation. The predicted SFG energy is obtained by squaring (25) and then integrating over time and space. We solve the Fourier-domain equation (49) and therefore integrate over energy and angle :

$$
\text { Energy }_{S F G}=\left(\chi_{N L} E_{\text {lighta }} E_{x a}\right)^{2} \iint H_{\text {wave eqn }}^{2} d \delta d \omega
$$

The corresponding expression for the input x-ray energy is :

$$
\text { Energy }_{\mathrm{x} \text {-ray }}=\mathrm{E}_{\mathrm{xa}}^{2} \iint \mathrm{F}_{\mathrm{x} \text {-ray }}^{2} \mathrm{~d} \delta \mathrm{d} \omega
$$

where $F_{x-\text { ray }}$ is a function specifying the (space/time) Gaussian beam profile of the input $\mathrm{x}$-ray field. The efficiency is the ratio of SFG to input X-ray energies :

$$
\eta \equiv \text { Energy }_{\mathrm{SFG}} / \text { Energy }_{\mathrm{x}-\text { ray }}=\left(\chi_{\mathrm{NL}} \mathrm{E}_{\text {lighta }}\right)^{2}\left(\iint \mathrm{H}_{\mathrm{wave}}{ }^{2} \mathrm{~d} \delta \mathrm{d} \omega\right) /\left(\int \mathrm{F}_{\mathrm{x} \text {-ray }}{ }^{2} \mathrm{~d} \delta \mathrm{d} \omega\right)
$$

The quantity $\chi_{\mathrm{NL}}$ is the sole unknown on the right hand side of (28) and it is determined by the measured efficiency $\eta$.

\subsection{Induced charge from nonlinear susceptibility}

Here we express the optically induced charge in terms of the nonlinear susceptibility. We rewrite equations (23) and (24) from above : 


\section{Supplemental Information}

$$
\begin{aligned}
& \omega_{\text {sum }} \mathrm{P}_{\mathrm{NL}}^{\mathrm{T}}=(\mathrm{e} / \mathrm{m})\left\{1 / \omega_{\mathrm{x}}\right\} \rho\left(\mathrm{E}_{\text {light }}^{\mathrm{G}}\right)^{\text {induced }} \mathrm{E}_{\mathrm{x}}^{\mathrm{T}} \\
& \mathrm{P}_{\mathrm{NL}}^{\mathrm{T}}\left(\mathbf{k}_{\text {sum }}\right) \equiv \chi_{\mathrm{NL}}(\mathbf{G}) \mathrm{E}_{\text {light }}{ }^{\mathrm{G}}\left(\mathbf{k}_{\mathrm{o}}\right) \mathrm{E}_{\mathrm{x}}^{\mathrm{T}}\left(\mathbf{k}_{\mathrm{x}}\right)
\end{aligned}
$$

Inserting (24) into (23) gives :

$$
\rho\left(E_{\text {light }}^{G}\right)^{\text {induced }}=\omega_{\mathrm{x}} \omega_{\text {sum }} \chi_{N L}(\mathbf{G}) E_{\text {light }}^{\mathrm{G}}\left(\mathbf{k}_{\mathrm{o}}\right)(\mathrm{m} / \mathrm{e})
$$

We rewrite (29) as :

$$
\rho\left(\mathbf{k}_{\mathrm{o}}+\mathbf{G}\right)^{\text {induced }}=\omega_{\mathrm{x}} \omega_{\text {sum }}\left(\chi_{\mathrm{NL}} \mathrm{E}_{\text {light }}^{\mathrm{G}}\right)(\mathrm{m} / \mathrm{e})
$$

With the nonlinear susceptibility $\chi_{\mathrm{NL}}\left(\mathbf{G}_{111}\right)=1.57 \times 10^{-14}$ esu and the optical field $\mathrm{E}_{\text {light }}{ }^{\mathrm{G}}$ $=9139.3$ statVolts $/ \mathrm{cm}\left(2.7 \times 10^{8} \mathrm{~V} / \mathrm{m}\right)$, we obtain a measured charge density of $\rho\left(\mathbf{k}_{0}+\mathbf{G}_{111}\right)^{\text {induced }}=4.0 \times 10^{10}$ statCoulombs $/ \mathrm{cm}^{3}\left(8.4 \times 10^{-5} \mathrm{e} / \AA^{3}\right)$.

\section{$\underline{3.3 \text { Induced Field from induced charge }}$}

Here the induced field $\left(\delta \mathbf{E}_{o}(\mathbf{r}, t)\right)$ is written in terms of the induced charge $\left(\delta \rho_{\mathrm{o}}(\mathbf{r}, \mathrm{t})\right)$. We start with the standard ${ }^{3}$ relationship between the induced charge and the associated induced field :

$$
\begin{aligned}
& \mathrm{D}=\mathbf{E}+4 \pi \mathbf{P} \\
& \nabla \cdot \mathrm{D}=4 \pi \rho_{\text {free }}=0 \\
& \nabla \cdot \delta \mathbf{E}_{\mathrm{o}}(\mathbf{r}, \mathrm{t})=4 \pi \delta \rho_{\mathrm{o}}(\mathbf{r}, \mathrm{t})
\end{aligned}
$$

where $\delta \rho_{o}(\mathbf{r}, \mathrm{t}) \equiv-\nabla \cdot \mathrm{P}$ and we note that (31) and (32) lead to (33). Expand $\delta \mathbf{E}_{\mathrm{o}}(\mathbf{r}, \mathrm{t})$ and $\delta \rho_{\mathrm{o}}(\mathbf{r}, \mathrm{t})$ in a Fourier series :

$$
\delta \rho_{\mathrm{o}}\left(\mathrm{r}, \omega_{\mathrm{o}}\right)=\Sigma_{\mathrm{Q}} \delta \rho_{\mathrm{o}}(\mathbf{Q}) \operatorname{Exp}[\mathrm{i} \mathbf{Q} \cdot \mathbf{r}] \operatorname{Exp}\left[-\mathrm{i} \omega_{\mathrm{o}} \mathrm{t}\right]
$$




$$
\delta \mathbf{E}_{\mathrm{o}}\left(\mathrm{r}, \omega_{\mathrm{o}}\right)=\Sigma_{\mathrm{Q}} \delta \mathbf{E}_{\mathrm{o}}(\mathbf{Q}) \operatorname{Exp}[\mathrm{i} \mathbf{Q} \cdot \mathbf{r}] \operatorname{Exp}\left[-i \omega_{\mathrm{o}} \mathrm{t}\right]
$$

Here, with $\mathbf{Q}=\mathbf{G}+\mathbf{k}_{0}, \delta \mathbf{E}_{0}(\mathbf{Q})$ is the $\mathbf{Q}^{\text {th }}$ Fourier component of the microscopic field arising in diamond due to application of the external field $\mathbf{E}_{\text {light }}\left(\mathbf{k}_{\mathrm{o}}, \omega_{\mathrm{o}}\right)^{3-6}$. Equations (33)-(35) lead to :

$$
\begin{aligned}
& \boldsymbol{\nabla} \cdot \Sigma_{\mathrm{Q}} \delta \mathbf{E}_{\mathrm{o}}(\mathbf{Q}) \operatorname{Exp}[\mathrm{i} \mathbf{Q} \cdot \mathbf{r}]=4 \pi \Sigma_{\mathrm{Q}} \delta \rho_{\mathrm{o}}(\mathbf{Q}) \operatorname{Exp}[\mathbf{i} \mathbf{Q} \cdot \mathbf{r}] \\
& \mathrm{iQ} \cdot \Sigma_{\mathrm{Q}} \delta \mathbf{E}_{\mathrm{o}}(\mathbf{Q}) \operatorname{Exp}[\mathrm{i} \mathbf{Q} \cdot \mathbf{r}]=4 \pi \Sigma_{\mathrm{Q}} \delta \rho_{\mathrm{o}}(\mathbf{Q}) \operatorname{Exp}[\mathrm{i} \mathbf{Q} \cdot \mathbf{r}]
\end{aligned}
$$

Noting that (37) holds term by term we obtain:

$$
\mathrm{i} \mathbf{Q} \cdot \delta \mathbf{E}_{\mathrm{o}}(\mathbf{Q})=4 \pi \delta \rho_{\mathrm{o}}(\mathbf{Q})
$$

Equation (38) indicates that the longitudinal (i.e. projection along $\mathbf{Q}$ ) component of the optically induced field $\delta \mathbf{E}_{\mathrm{o}}(\mathbf{Q})$ is determined by the measured quantity $\delta \rho_{\mathrm{o}}(\mathbf{Q})$; $\mathbf{Q}=\mathbf{G}_{111}+\mathbf{k}_{\mathrm{o}}$ for the current experiment. Additionally, it can be shown ${ }^{4-6}$ that the transverse component is of order $[\mathrm{q} /|\mathbf{q}+\mathbf{G}|]^{2}$ times the longitudinal component so that for $\mathbf{q}=\mathbf{k}_{\mathrm{o}},\left[\mathbf{q} /\left|\mathbf{q}+\mathbf{G}_{111}\right|\right]^{2} \sim 10^{-7}$ and $\delta \mathbf{E}_{\mathrm{o}}(\mathbf{Q})$ is, to accuracy much better than $1 \%$, equal to the longitudinal component specified by (38).

The macroscopic component of the internal field, $\delta \mathbf{E}_{0}\left(\mathbf{Q}=\mathbf{0}+\mathbf{k}_{\mathbf{0}}\right) \equiv \mathbf{E}_{\mathbf{k}}$, is related to the vacuum amplitude (less surface reflectivity loss) of the optical field $\left(\mathbf{E}_{\text {light }}\right)$ as $^{3}$ :

$$
\mathbf{E}_{\mathbf{k o}}=\{3 /(2+\varepsilon)\} \mathbf{E}_{\text {light }}
$$

Here $\varepsilon=5.8$ is the static, long wavelength dielectric constant for diamond. With $\mathrm{E}_{\text {light }}{ }^{\mathrm{Q}}$ representing the component of $\mathbf{E}_{\text {light }}$ along $\mathbf{Q}$, and $\mathrm{E}_{\mathrm{ko}}{ }^{\mathrm{Q}}$ representing the component of $\mathbf{E}_{\text {ko }}$ along $\mathbf{Q}$, the experimental value is $\mathrm{E}_{\text {light }}{ }^{\mathrm{Q}}=9139.3$ statVolts $/ \mathrm{cm}$. Using the 


\section{Supplemental Information}

measured value $\delta \rho_{\mathrm{o}}\left(\mathbf{k}_{\mathrm{o}}+\mathbf{G}_{111}\right)=4.0 \times 10^{10}$ statCoulombs $/ \mathrm{cm}^{3}\left(8.4 \times 10^{-5} \mathrm{e} / \AA^{3}\right)$, equations (38) and (39) indicate :

$$
\begin{aligned}
& \left|\delta \mathbf{E}_{\mathrm{o}}(\mathbf{Q})\right| / \mathrm{E}_{\mathbf{k o}}{ }^{\mathrm{Q}}=0.47 \\
& \left|\delta \mathbf{E}_{\mathrm{o}}(\mathbf{Q})\right| / \mathrm{E}_{\text {light }}{ }^{\mathrm{Q}}=0.18
\end{aligned}
$$

Equations (40) and (41) specify the magnitude of the optically induced field, $\left|\delta \mathbf{E}_{\mathrm{o}}(\mathbf{Q})\right|$ (where $\mathbf{Q}=\mathbf{k}_{\mathrm{o}}+\mathbf{G}_{111}$ ), as a fraction of the corresponding longitudinal components of the macroscopic internal optical field (40) and the unscreened external optical field (41) respectively. The uncertainty in $\delta \rho_{\mathrm{o}}(\mathbf{Q}), \chi_{\mathrm{NL}}\left(\mathbf{G}_{111}\right)$, and $\delta \mathbf{E}_{\mathrm{o}}(\mathbf{Q})$ is $\sim 40 \%$, determined by the estimated $\mathrm{x} 2$ uncertainty in the measured efficiency.

\section{Derivation of Wave Equation}

Generation of an electric field (E') at the sum frequency is described by the time and space dependent wave equation :

$$
\nabla^{2} \mathbf{E}^{\prime}-\left(1 / \mathrm{c}^{2}\right) \mathrm{d}^{2} \mathbf{E}^{\prime} / \mathrm{dt}^{2}-(2 \beta / \mathrm{c}) \mathrm{d} \mathbf{E}, / \mathrm{dt}=\left(4 \pi / \mathrm{c}^{2}\right) \mathrm{d}^{2} \mathbf{P}^{\prime}{ }_{\mathrm{NL}} / \mathrm{dt}^{2}
$$

Here $\beta$ is the $\mathbf{x}$-ray absorption coefficient and $\mathbf{P}^{\prime}{ }_{\mathbf{N L}}$ is the induced nonlinear polarization at the sum frequency. The z-axis is the inward facing surface normal and the $\mathrm{x}$-axis is parallel to the sample surface. Phasematching is insensitive to small out-of-diffractionplane rotations and we determine that to good approximation one can ignore the out-ofplane (y) axis. Similarly, we assume that transverse angular spread, group velocity dispersion, and higher order dispersion are negligible. The field and polarization are written as a product of a plane wave carrier term and a slowly varying envelope :

$$
\mathbf{E}^{\prime}=\mathbf{E}(\mathbf{r}, \mathrm{t}) \operatorname{Exp}\left[-\mathrm{i}\left(\omega_{\mathrm{s}} \mathrm{t}-\mathbf{k}_{\mathbf{s}} \cdot \mathbf{r}\right)\right] \quad \mathbf{P}^{\prime}{ }_{\mathbf{N L}}=\mathbf{P}_{\mathrm{NL}}(\mathbf{r}, \mathrm{t}) \operatorname{Exp}\left[-\mathrm{i}\left(\omega_{\mathrm{s}} \mathrm{t}-\mathbf{k}_{\mathbf{s}} \cdot \mathbf{r}\right)\right]
$$




\section{Supplemental Information}

Inserting these forms into the wave equation and using the slowly varying envelope approximation $^{29}$ to neglect second derivatives of the field envelope and both first and second derivatives of polarization envelope, one obtains the following equation relating the field and polarization envelopes :

$$
\begin{aligned}
& \cos \left(\theta_{\mathrm{s}}\right) \mathrm{d} \mathbf{E} / \mathrm{dz}+\sin \left(\theta_{\mathrm{s}}\right) \mathrm{d} \mathbf{E} / \mathrm{dx}+(1 / \mathrm{c}) \mathrm{d} \mathbf{E} / \mathrm{dt}+\beta \mathbf{E}=\mathrm{i} \mathbf{P}_{\mathrm{NL}}\left((2 \pi)^{2} / \lambda\right) \\
& \cos \left(\theta_{\mathrm{s}}\right) \mathrm{d} \mathbf{E} / \mathrm{dz}+\sin \left(\theta_{\mathrm{s}}\right) \mathrm{d} \mathbf{E} / \mathrm{dx}+(1 / \mathrm{c}) \mathrm{d} \mathbf{E} / \mathrm{dt}+\beta \mathbf{E}=(-2 \pi / \mathrm{c}) \mathbf{J}_{\mathrm{NL}}
\end{aligned}
$$

Here $\lambda$ is the SFG wavelength and $\mathbf{J}_{\mathbf{N L}}$ is the sum frequency current (envelope), related to the nonlinear polarization by (22). In (44) $\mathbf{E}=\mathbf{E}(\mathrm{z}, \mathrm{x}, \mathrm{t})$. The two dimensional Fourier transform (time and transverse spatial co-ordinate $\mathrm{x}$ ), $\mathrm{FT}_{\mathrm{x}, \mathrm{t}}\{\}$, of (44) gives :

$$
\cos \left(\theta_{\mathrm{s}}\right) \mathrm{d} \mathbf{E} / \mathrm{dz}-\sin \left(\theta_{\mathrm{s}}\right)\left(\mathrm{i} \mathrm{k}_{\mathrm{x}}\right) \mathbf{E}-(\mathrm{i} \omega / \mathrm{c}) \mathbf{E}+\beta \mathbf{E}=\mathrm{FT}_{\mathrm{x}, \mathrm{t}}\left\{\mathrm{i} \mathbf{P}_{\mathrm{NL}}\left((2 \pi)^{2} / \lambda\right)\right\}
$$

In (45) $\mathbf{E}=\mathbf{E}\left(\mathrm{z}, \mathrm{k}_{\mathrm{x}}, \omega\right)$. We next define the total transverse wave vector $\mathrm{k}_{\mathrm{x}, \mathrm{TOT}}$ as the sum of carrier and envelope wave vectors :

$$
\mathrm{k}_{\mathrm{x}, \mathrm{TOT}}=\mathrm{k}_{\mathrm{x}, \mathrm{env}}+\mathrm{k}_{\mathrm{x}, \text { carrier }}
$$

The total transverse wave vector $\left(\mathrm{k}_{\mathrm{x}, \mathrm{TOT}}\right)$ is equal to the projection of the total wave vector $\left(\mathbf{k}_{\mathrm{s}}\right)$ on to the $\mathrm{x}$-axis. With $\theta_{\mathrm{s}}$ defined as the angle $\mathbf{k}_{\mathrm{s}}$ makes with the $\mathrm{z}$-axis, $\mathrm{k}_{\mathrm{x}, \text { TOT }}$ $=\mathrm{k}_{\mathrm{s}} \sin \left(\theta_{\mathrm{s}}\right)$. We write $\theta_{\mathrm{s}}$ as the sum of a 'perfect phasematching' angle $\theta_{\mathrm{so}}$ and a deviation angle $\delta$ :

$$
\begin{gathered}
\mathrm{k}_{\mathrm{x}, \mathrm{TOT}}=\mathrm{k}_{\mathrm{sfg}} \sin \left(\theta_{\mathrm{s}}\right)=\mathrm{k}_{\mathrm{sfg}} \sin \left(\theta_{\mathrm{so}}+\delta\right)=\mathrm{k}_{\mathrm{sfg}}\left\{\sin \left(\theta_{\mathrm{so}}\right) \cos (\delta)+\sin (\delta) \cos \left(\theta_{\mathrm{so}}\right)\right\} \\
\mathrm{k}_{\mathrm{x}, \mathrm{TOT}} \approx\left\{\mathrm{k}_{\mathrm{sfg}} \sin \left(\theta_{\mathrm{so}}\right)+\delta \mathrm{k}_{\mathrm{sfg}} \cos \left(\theta_{\mathrm{so}}\right)\right\}
\end{gathered}
$$




\section{Supplemental Information}

The carrier wave vector is defined to be at the 'perfect' phasematching angle while the

envelope wave vector accounts for any deviation, $\mathrm{k}_{\mathrm{x}, \mathrm{carrier}} \equiv \mathrm{k}_{\mathrm{sfg}} \sin \left(\theta_{\mathrm{so}}\right)$. Accordingly :

$$
\mathrm{k}_{\mathrm{x}, \text { env }}=\mathrm{k}_{\mathrm{x}, \mathrm{TOT}}-\mathrm{k}_{\mathrm{x}, \text { carrier }}=\delta \mathrm{k}_{\mathrm{sfg}} \cos \left(\theta_{\mathrm{so}}\right)
$$

We note that $\mathrm{k}_{\mathrm{x}}$ in (45) represents the transverse wave vector of the envelope $\left(\mathrm{k}_{\mathrm{x}, \mathrm{env}}\right)$ so that inserting (48) into (45) leads to :

$$
\begin{aligned}
& \cos \left(\theta_{\mathrm{so}}\right) \mathrm{d} \mathbf{E} / \mathrm{dz}-\mathrm{i} \sin \left(\theta_{\mathrm{so}}\right) \cos \left(\theta_{\mathrm{so}}\right) \mathrm{k}_{\mathrm{s}} \delta \mathbf{E}-(\mathrm{i} \omega / \mathrm{c}) \mathbf{E}+\beta \mathbf{E}=\mathrm{FT}_{\mathrm{x}, \mathrm{t}}\left\{\mathrm{i} \mathbf{P}_{\mathrm{NL}}\left((2 \pi)^{2} / \lambda\right)\right\} \\
& \cos \left(\theta_{\mathrm{so}}\right) \mathrm{d} \mathbf{E} / \mathrm{dz}-\mathrm{i} \sin \left(\theta_{\mathrm{so}}\right) \cos \left(\theta_{\mathrm{so}}\right) \mathrm{k}_{\mathrm{s}} \delta \mathbf{E}-(\mathrm{i} \omega / \mathrm{c}) \mathbf{E}+\beta \mathbf{E}=\mathrm{FT}_{\mathrm{x}, \mathrm{t}}\left\{(-2 \pi / \mathrm{c}) \mathrm{J}_{\mathrm{NL}}\right\}
\end{aligned}
$$

Equation (49b) is obtained using (22) and equations (49) are solved subject to the condition that the input SFG field is zero. The angle $\theta_{\mathrm{so}}=57.3$ degrees is specified by energy and momentum conservation and knowledge of the (input) x-ray/optical crossing angle in vacuum, 14.3 degrees with the x-ray and optical beams on opposite sides of the sample surface normal.

\section{Relative nonlinear current amplitudes}

Here we show that of the six scattering terms contributing to x-ray/optical SFG, the SFG power is dominated by inelastic x-ray scatter off of optically induced charge oscillations. We also show that the nonlinear current from x-ray/optical SFG should be significantly larger than that from either x-ray/euv or x-ray/x-ray SFG.

There are three types of sum frequency currents ${ }^{29}$. First, a so-called Doppler current representing inelastic scatter by one field off of charge oscillations induced by a second field. Next, a contribution from the familiar $\mathbf{v x B}$ Lorentz force term. Third, a socalled displacement current arising from the fact that the displacement of an electron by one field causes it to sample a different spatial region of a second field. Each of the 


\section{Supplemental Information}

three types of currents has two contributions corresponding to the x-ray and optical fields switching roles. The six terms contributing to x-ray/optical SFG are given as (see "Derivation of nonlinear current terms" below) :

$$
\begin{aligned}
& \mathrm{J}_{\mathrm{a}}{ }^{\mathrm{T}} \text { Doppler }(\mathrm{o}, \mathrm{x})=\mathrm{i}(\mathrm{e} / 2 \mathrm{~m}) \mathrm{D} \omega_{\mathrm{x}} \rho_{\mathrm{o}}{ }^{(1)} \boldsymbol{\varepsilon}_{\mathrm{SFG}} \cdot \mathbf{E}_{\mathrm{x}} \\
& \mathrm{J}_{\mathrm{a}}{ }^{\mathrm{T}} \text { Doppler }(\mathrm{x}, \mathrm{o})=\mathrm{i}(\mathrm{e} / 2 \mathrm{~m}) \mathrm{D} \omega_{\mathrm{o}} \rho_{\mathrm{x}}{ }^{(1)} \boldsymbol{\varepsilon}_{\mathrm{SFG}} \cdot \mathbf{E}_{\mathrm{o}} \\
& \mathrm{J}_{\mathrm{a}}{ }^{\mathrm{T}} \text { Displacement }(\mathrm{o}, \mathrm{x})=-\rho^{(\mathrm{u})}\left[\mathrm{e}^{2} /\left(4 \mathrm{~m}^{2} \omega_{\text {sum }}\right)\right] \mathrm{D} \omega_{\mathrm{o}} \mathrm{D} \omega_{\mathrm{x}}\left(\mathbf{E}_{\mathrm{o}} \cdot \mathbf{k}_{\mathrm{x}}\right) \boldsymbol{\varepsilon}_{\mathrm{SFG}} \cdot \mathbf{E}_{\mathrm{x}} \\
& \mathrm{J}_{\mathrm{a}}{ }^{\mathrm{T}} \text { Displacement }(\mathrm{x}, \mathrm{o})=-\rho^{(\mathrm{u})}\left[\mathrm{e}^{2} /\left(4 \mathrm{~m}^{2} \omega_{\text {sum }}\right)\right] \mathrm{D} \omega_{\mathrm{x}} \mathrm{D} \omega_{\mathrm{o}}\left(\mathbf{E}_{\mathrm{x}} \cdot \mathbf{k}_{\mathrm{o}}\right) \boldsymbol{\varepsilon}_{\mathrm{SFG}} \cdot \mathbf{E}_{\mathrm{o}} \\
& \mathrm{J}_{\mathrm{a}}{ }^{\mathrm{T}} \text { Lorentz }(\mathrm{o}, \mathrm{x})=\rho^{(\mathrm{u})}\left[\mathrm{e}^{2} /\left(2 \mathrm{~m}^{2} \omega_{\text {sum }}\right)\right]\left(\mathrm{D} \omega_{\mathrm{o}} / \omega_{\mathrm{x}}\right) \mathrm{E}_{\mathrm{o}} \mathrm{E}_{\mathrm{x}} \mathrm{k}_{\mathrm{x}} \boldsymbol{\varepsilon}_{\mathrm{SFG}} \cdot \mathbf{k}_{\mathbf{o}} /\left|\mathbf{k}_{\mathbf{o}}\right| \\
& \mathrm{J}_{\mathrm{a}}^{\mathrm{T} \text { Lorentz }}(\mathrm{x}, \mathrm{o})=\rho^{(\mathrm{u})}\left[\mathrm{e}^{2} /\left(2 \mathrm{~m}^{2} \omega_{\text {sum }}\right)\right]\left(\mathrm{D} \omega_{\mathrm{x}} / \omega_{\mathbf{o}}\right) \mathrm{E}_{\mathrm{o}} \mathrm{E}_{\mathrm{x}} \mathrm{k}_{\mathbf{o}} \boldsymbol{\varepsilon}_{\mathrm{SFG}} \cdot \mathbf{k}_{\mathrm{x}} /\left|\mathbf{k}_{\mathrm{x}}\right| \\
& D \omega_{j} \equiv \omega_{j} /\left(\omega_{b}^{2}-\omega_{j}^{2}\right)
\end{aligned}
$$

The subscripts ' $o$ ' and ' $x$ ' refer to optical and $x$-ray fields respectively, $\omega_{\text {sum }}$ is the sum frequency $\omega_{\mathrm{x}}+\omega_{0}, \boldsymbol{\varepsilon}_{\mathrm{SFG}}$ is the sum frequency polarization vector, and $h \omega_{\mathrm{b}} / 2 \pi$ is equal to the bandgap in diamond $(\sim 5.5 \mathrm{eV})$. In equations $(50) \rho_{\mathrm{i}}^{(1)}$ is the first order charge density induced by field ' $i$ ' and $\rho^{(\mathrm{u})}$ is the unperturbed charge density.

A sum frequency polarization is produced because an electron is driven simultaneously by both fields. To the extent that an electron is negligibly perturbed by either field, then the contribution to the sum frequency from this electron is negligible. As the x-ray energy is large compared to binding energies in diamond, all electrons in diamond respond similarly to x-rays. This is not the case for light : optically polarizable charge contributes while charge with negligible optical polarizability does not. Each nonlinear current term is linearly proportional to this optically polarizable charge component $\left(\rho_{\text {optical }}\right)$ : the unperturbed charge density is equal to this polarizable 


\section{Supplemental Information}

component $\left(\rho^{(\mathrm{u})}=\rho_{\text {optical }}\right)$ and the induced charge $\left(\rho_{\mathrm{i}}^{(1)}\right)$ is linearly proportional to $\rho_{\text {optical }}$ since both fields must drive this same charge component. The density functional theory calculations described below indicate that $\rho_{\text {optical }}$ is, for the current purposes, well approximated by the bond-charge distribution and the currents below assume $\rho_{\text {optical }}=\rho_{\text {bond. }}$. The relative current amplitudes, however, are independent of this choice. We quote relative amplitudes by defining $\mathrm{J}^{\mathrm{T}}$ Doppler $(\mathrm{o}, \mathrm{x})=1$. We further assume that the x-ray and optical beams are collinear and set all angular factors $\cos (\theta)$ equal to one. Evaluating equations (50) leads to the following relative current amplitudes :

$$
\begin{aligned}
& \mathrm{J}_{\text {Doppler }}^{\mathrm{T}}(\mathrm{o}, \mathrm{x})=1 \quad \text { (optically induced charge) } \\
& \mathrm{J}_{\text {Lorentz }}^{\mathrm{T}}(\mathrm{o}, \mathrm{x})=2.6 \times 10^{-4} \\
& \mathrm{~J}_{\text {Doppler }}^{\mathrm{T}}(\mathrm{x}, \mathrm{o})=1.9 \times 10^{-4} \\
& \mathrm{~J}_{\text {Displacement }}^{\mathrm{T}}(\mathrm{o}, \mathrm{x})=1.3 \times 10^{-4} \\
& \mathrm{~J}_{\text {Lorentz }}^{\mathrm{T}}(\mathrm{x}, \mathrm{o})=1.4 \times 10^{-6} \\
& \mathrm{~J}_{\text {Displacement }}^{\mathrm{T}}(\mathrm{x}, \mathrm{o})=6 \times 10^{-8}
\end{aligned}
$$

We see that the Doppler term, $\mathrm{J}^{\mathrm{T}}$ Doppler $(0, \mathrm{x})$, corresponding to optically induced charge oscillations dominates. Our measurements indicate that the harmonically bound electron model overestimates the induced charge by a factor of $\sim 12$; the model therefore also overestimates the optical polarizability and induced first order velocity by this factor. This over-estimation does not change the predicted relative current strengths since each current term contains either an optical first order velocity or an optical polarizability.

The same set of equations (50) can be used to estimate relative current magnitudes for different $\mathrm{x}$-ray summing processes. Since both the polarizability $\left(1 / \omega^{2}\right)$ and the electric field induced velocity $(1 / \omega)$ decrease with frequency, the second order 


\section{Supplemental Information}

current for $\mathrm{x}$-ray/optical mixing is significantly larger than that for $\mathrm{x}$-ray/x-ray or $\mathrm{x}$ ray/euv mixing. To illustrate, consider a free electron gas interacting with $\mathrm{x}$-rays (8 $\mathrm{keV})$ and euv radiation $(100 \mathrm{eV})$ or optical radiation $(800 \mathrm{~nm} ; 1.55 \mathrm{eV})$. For simplicity we assume that the Doppler-term induced charge is that for a free electron :

$$
\rho_{\mathbf{Q}}\left(\mathbf{E}_{\mathrm{i}}\right)^{\text {induced }}=\rho^{(\mathrm{u})}\left\{\mathbf{Q} \cdot \mathbf{E}_{\mathrm{i}} / \mathrm{e}\right\} \alpha_{\text {free }}\left(\omega_{\mathrm{i}}\right)=\rho^{(\mathrm{u})}\left\{\mathbf{G} \cdot \mathbf{E}_{\mathrm{i}} / \mathrm{e}\right\}\left(\mathrm{e}^{2} / \mathrm{m} \omega_{\mathrm{i}}^{2}\right)
$$

Given input fields ' 1 ' and ' 2 ', the Doppler sum frequency current scales as $\left\{\omega_{1} \omega_{2}{ }^{2}\right\}^{-1}$ while the Displacement and Lorentz currents scale as $\left\{\omega_{1} \omega_{\text {sum }}\right\}^{-1}$. Within any given SFG process, i.e. a fixed set of frequencies $\left(\omega_{1}, \omega_{2}\right)$, the Doppler current dominates so that the relative current amplitude for various frequency pairs of interest can be obtained simply from the Doppler scaling, $\left\{\omega_{1} \omega_{2}^{2}\right\}^{-1}$. More generally we find that the relative magnitudes of the nonlinear currents for $\mathrm{x}$-ray/x-ray, $\mathrm{x}$-ray/euv, and $\mathrm{x}$-ray/optical summing processes are :

$$
\begin{aligned}
& \mathrm{J}^{\mathrm{T}} \text { Doppler }(\mathrm{o}, \mathrm{x})=1 \quad \text { (optically induced charge) } \\
& \mathrm{J}_{\text {Lorentz }}^{\mathrm{T}}(\mathrm{o}, \mathrm{x})=2.6 \times 10^{-4} \\
& \mathrm{~J}^{\mathrm{T}}{ }_{\text {Doppler }}(\mathrm{euv}, \mathrm{x})=2.4 \times 10^{-4} \\
& \mathrm{~J}^{\mathrm{T}}{ }_{\text {Doppler }}(\mathrm{x}, \mathrm{o})=1.9 \times 10^{-4} \\
& \mathrm{~J}^{\mathrm{T}}{ }_{\text {Displacement }}(\mathrm{o}, \mathrm{x})=1.3 \times 10^{-4} \\
& \mathrm{~J}^{\mathrm{T}}{ }_{\text {Lorentz }}(\mathrm{euv}, \mathrm{x})=3.9 \times 10^{-6} \\
& \mathrm{~J}^{\mathrm{T}}{ }_{\text {Doppler }}(\mathrm{x}, \text { euv })=3.0 \times 10^{-6} \\
& \mathrm{~J}^{\mathrm{T}}{ }_{\text {Displacement }}(\mathrm{euv}, \mathrm{x})=2.0 \times 10^{-6} \\
& \mathrm{~J}^{\mathrm{T}}{ }_{\text {Lorentz }}(\mathrm{x}, \mathrm{o})=1.2 \times 10^{-7}
\end{aligned}
$$




\section{Supplemental Information}

$$
\begin{aligned}
& \mathrm{J}_{\text {Displacement }}^{\mathrm{T}}(\mathrm{x}, \mathrm{o})=6 \times 10^{-8} \\
& \mathrm{~J}_{\text {Lorentz }}^{\mathrm{T}}(\mathrm{x}, \mathrm{euv})=4.9 \times 10^{-8} \\
& \mathrm{~J}^{\mathrm{T}}{ }_{\text {Doppler }}(\mathrm{x}, \mathrm{X})=3.8 \times 10^{-8} \\
& \mathrm{~J}^{\mathrm{T}} \text { Displacement }(\mathrm{x}, \mathrm{euv})=2.5 \times 10^{-8} \\
& \mathrm{~J}_{\text {Lorentz }}^{\mathrm{T}}(\mathrm{x}, \mathrm{x})=2.5 \times 10^{-8} \\
& \mathrm{~J}^{\mathrm{T}}{ }_{\text {Displacement }}(\mathrm{x}, \mathrm{x})=1.2 \times 10^{-8}
\end{aligned}
$$

The current density for x-ray/optical SFG is therefore considerably larger than that for x-ray/euv SFG and both are significantly stronger than x-ray/x-ray SFG. The SFG efficiency scales as the square of these current density magnitudes. An accurate estimate of the SFG efficiency requires detailed consideration to account for factors such as phase matching, photoabsorption, and the fact that different components of the charge density can contribute to the different scattering processes. Nonetheless, these additional considerations should not change the conclusion that significantly lower efficiency can be expected when moving from x-ray/optical to analogous all-high-frequency processes.

\section{Derivation of nonlinear current terms}

We start by writing the wave equation :

$$
\nabla^{2} \mathbf{E}_{\text {real }}-\left(\varepsilon / \mathrm{c}^{2}\right) \mathrm{d}^{2} \mathbf{E}_{\text {real }} / \mathrm{dt}^{2}=\left(4 \pi / \mathrm{c}^{2}\right) \mathrm{d}^{2} \mathbf{P}_{\text {real }} \mathrm{NL} / \mathrm{dt}^{2}=\left(4 \pi / \mathrm{c}^{2}\right) \mathrm{d} \mathbf{J}_{\text {real }} / \mathrm{dt}
$$

For the purposes of this derivation we ignore absorption and the subscripts indicate that we consider real fields in (52). We next write the real fields using complex notation :

$$
\begin{aligned}
& \mathbf{E}_{\text {real }}=\mathbf{E}_{\mathrm{r}}(\mathbf{r}, \mathrm{t}) \operatorname{Cos}\left[\mathrm{i}\left(\mathbf{k}_{\mathrm{s}} \cdot \mathbf{r}-\omega_{\mathrm{s}} \mathrm{t}\right)\right] \equiv 1 / 2\left\{\mathbf{E}_{\mathrm{c}}+\text { c.c. }\right\} \\
& \mathbf{J}_{\text {real }}=\mathbf{J}_{\mathrm{r}}(\mathbf{r}, \mathrm{t}) \operatorname{Cos}\left[\mathrm{i}\left(\mathbf{k}_{\mathrm{s}} \cdot \mathbf{r}-\omega_{\mathrm{s}} \mathrm{t}\right)\right] \equiv \mathbf{J}_{\mathrm{a}}+\text { c.c. }
\end{aligned}
$$




\section{Supplemental Information}

where the right most terms define $\mathbf{E}_{\mathrm{c}}$ and $\mathbf{J}_{\mathrm{a}}$ and 'c.c.' stands for complex conjugate. Inserting these forms into (52) we obtain :

$$
\nabla^{2} 1 / 2\left\{\mathbf{E}_{\mathrm{c}}+\text { c.c. }\right\}-\left(\varepsilon / \mathrm{c}^{2}\right) \mathrm{d}^{2} 1 / 2\left\{\mathbf{E}_{\mathrm{c}}+\text { c.c. }\right\} / \mathrm{dt}^{2}=\left(4 \pi / \mathrm{c}^{2}\right) \mathrm{d}\left\{\mathbf{J}_{\mathrm{a}}+\text { c.c. }\right\} / \mathrm{dt}
$$

We multiple both sides by 2 and separate into two equations, one the complex conjugate of the other :

$$
\begin{aligned}
& \nabla^{2} \mathbf{E}_{\mathrm{c}}-\left(\varepsilon / \mathrm{c}^{2}\right) \mathrm{d}^{2} \mathbf{E}_{\mathrm{c}} / \mathrm{dt}^{2}=\left(4 \pi / \mathrm{c}^{2}\right) \mathrm{d}\left(2 \mathbf{J}_{\mathrm{a}}\right) / \mathrm{dt} \\
& \boldsymbol{\nabla}^{2} \mathbf{E}_{\mathrm{c}}{ }^{*}-\left(\varepsilon / \mathrm{c}^{2}\right) \mathrm{d}^{2} \mathbf{E}_{\mathrm{c}}{ }^{*} / \mathrm{dt}^{2}=\left(4 \pi / \mathrm{c}^{2}\right) \mathrm{d}\left(2 \mathbf{J}_{\mathrm{a}}{ }^{*}\right) / \mathrm{dt}
\end{aligned}
$$

We no longer consider equation (56b) since it provides redundant information. We will derive an expression for the nonlinear current using fields written in the form given by (53). Wave equation (56a) shows that the nonlinear current source term, the quantity on the right hand side of the wave equation, is $\mathbf{J}_{\mathrm{NL}}=2 \mathbf{J}_{\mathrm{a}}$; i.e. the nonlinear current entering the wave equation is twice the current obtained from fields written in form (53). We next derive an expression for $\mathbf{J}^{(2)}\left(\omega_{1}+\omega_{2}\right)$ using fields written in form (53).

We essentially follow the derivation in [29]. Two types of terms contribute to the second order current density : the zeroth order (i.e. unperturbed) charge density $\left(\rho^{(u)}\right)$ times a second order velocity and a first order (i.e. induced) charge density times a first order velocity. The general expression for $\mathbf{J}^{(2)}$ is :

$$
\mathbf{J}^{(2)}=\rho^{(1)} \mathbf{v}^{(1)}+\rho^{(\mathrm{u})} \mathbf{v}^{(2)}
$$

We begin by treating the electrons as bound harmonic oscillators subject to the Lorentz force : 


\section{Supplemental Information}

$$
\mathrm{d} \mathbf{v} / \mathrm{dt}=-\omega_{\mathrm{b}}^{2} \mathrm{x}-(\mathrm{e} / \mathrm{m})\{\mathbf{E}+\mathbf{v} / \mathrm{cx} \mathbf{B}\}=\delta \mathbf{v} / \delta \mathrm{t}+(\mathbf{v} \cdot \boldsymbol{\nabla}) \mathbf{v}
$$

where $h \omega_{\mathrm{b}} / 2 \pi$ is the binding energy, $\mathrm{x}$ is a spatial coordinate, and $\delta$ indicates partial derivative. We rewrite (58) ignoring the second order $\mathbf{v x B}$ and $(\mathbf{v} \cdot \nabla) \mathbf{v}$ terms to determine the first order velocity :

$$
\delta \mathbf{v}^{(1)} / \delta \mathrm{t}=-\omega_{\mathrm{b}}^{2} \mathrm{x}-(\mathrm{e} / \mathrm{m}) \mathbf{E}
$$

We next assume a specific form for $\mathrm{E}$ :

$$
\begin{gathered}
\mathbf{E}_{\text {Total }}=\mathbf{E}_{\text {real }, 1} \cos \left(\mathbf{k}_{1} \cdot \mathbf{r}-\omega_{1} \mathrm{t}\right)+\mathbf{E}_{\text {real }, 2} \cos \left(\mathbf{k}_{2} \cdot \mathbf{r}-\omega_{2} \mathrm{t}\right) \\
\mathbf{E}_{\text {Total }}=\left\{1 / 2 \mathbf{E}_{\mathrm{c} 1} \operatorname{Exp}\left[\mathbf{k}_{1} \cdot \mathbf{r}-\omega_{1} \mathrm{t}\right]+\text { c.c. }\right\}+\left\{1 / 2 \mathbf{E}_{\mathrm{c} 2} \operatorname{Exp}\left[\mathbf{k}_{2} \cdot \mathbf{r}-\omega_{2} \mathrm{t}\right]+\text { c.c. }\right\}
\end{gathered}
$$

where the subscript 'Total' refers to the sum of input fields ' 1 ' and ' 2 '. From (60a) and (60b) we see $\mathbf{E}_{\mathrm{cj}}=\mathbf{E}_{\text {real }, \mathrm{j}}$ and we simplify the subscript to $\mathbf{E}_{\mathrm{j}}$. Inserting (60) into (59):

$$
\delta \mathbf{v}^{(1)} / \delta \mathrm{t}=-\omega_{\mathrm{b}}{ }^{2} \mathrm{x}-(\mathrm{e} / 2 \mathrm{~m})\left[\left\{\mathbf{E}_{1} \operatorname{Exp}\left[\left[\mathbf{k}_{1} \cdot \mathbf{r}-\omega_{1} \mathrm{t}\right]+\text { c.c. }\right\}+\left\{\mathbf{E}_{2} \operatorname{Exp}\left[\mathbf{k}_{2} \cdot \mathbf{r}-\omega_{2} \mathrm{t}\right]+\text { c.c. }\right\}\right](61)\right.
$$

Equation (61) is a linear equation with four field terms $\left(\mathbf{E}_{1}, \mathbf{E}_{2},+\right.$ c.c. terms $)$ as 'sources'; $\mathbf{v}^{(1)}$ will be the sum of four terms, two c.c. terms and two terms $(j=1,2)$ given as :

$$
\mathbf{v}_{\mathrm{j}}^{(1)}=\mathrm{i}(\mathrm{e} / 2 \mathrm{~m})\left(\omega_{\mathrm{j}} /\left(\omega_{\mathrm{b}}{ }^{2}-\omega_{\mathrm{j}}^{2}\right)\right) \mathbf{E}_{\mathrm{j}} \operatorname{Exp}\left[\mathbf{k}_{\mathrm{j}} \cdot \mathbf{r}-\omega_{\mathrm{j}} \mathrm{t}\right]
$$

We next obtain an expression for $\mathbf{v}^{(2)}$ by neglecting the first order terms $\omega_{b}{ }^{2} \mathrm{x}$ and $(\mathrm{e} / \mathrm{m}) \mathbf{E}$ in $(58):$

$$
\delta \mathbf{v}^{(2)} / \delta \mathrm{t}=-(\mathrm{e} / \mathrm{mc}) \mathbf{v}^{(1)} \mathrm{xB}-\left(\mathbf{v}^{(1)} \cdot \nabla\right) \mathbf{v}^{(1)}
$$




\section{Supplemental Information}

We consider only the $\mathbf{v}^{(2)}$ terms contributing to $\omega_{1}+\omega_{2}$ and choose to separate this version of (63) into four equations (two plus c.c. terms):

$$
\begin{aligned}
& \delta \mathbf{v}_{1,2}{ }^{(2)} / \delta \mathrm{t}=-\mathrm{i} \omega_{\text {sum }} \mathbf{v}_{1,2}{ }^{(2)}=-(\mathrm{e} / \mathrm{mc}) \mathbf{v}_{1}{ }^{(1)} \mathrm{x} \mathbf{B}_{2}-\left(\mathbf{v}_{1}{ }^{(1)} \cdot \nabla\right) \mathbf{v}_{2}{ }^{(1)} \\
& \delta \mathbf{v}_{2,1}{ }^{(2)} / \delta \mathrm{t}=-\mathrm{i} \omega_{\text {sum }} \mathbf{v}_{2,1}{ }^{(2)}=-(\mathrm{e} / \mathrm{mc}) \mathbf{v}_{2}{ }^{(1)} \mathrm{x} \mathbf{B}_{1}-\left(\mathbf{v}_{2}{ }^{(1)} \cdot \nabla\right) \mathbf{v}_{1}{ }^{(1)} \\
& \delta \mathbf{v}_{1,2}{ }^{(2)^{*}} / \delta \mathrm{t}=+\mathrm{i} \omega_{\text {sum }} \mathbf{v}_{1,2}{ }^{(2)^{*}}=-(\mathrm{e} / \mathrm{mc}) \mathbf{v}_{1}{ }^{(1)^{*}} \mathrm{xB}_{2}{ }^{*}-\left(\mathbf{v}_{1}{ }^{(1)^{*}} \cdot \nabla\right) \mathbf{v}_{2}{ }^{(1)^{*}} \\
& \delta \mathbf{v}_{2,1}{ }^{(2)^{*}} / \delta \mathrm{t}=+\mathrm{i} \omega_{\text {sum }} \mathbf{v}_{2,1}{ }^{(2)^{*}}=-(\mathrm{e} / \mathrm{mc}) \mathbf{v}_{2}{ }^{(1)^{*}}{ }^{*} \mathbf{B}_{1}{ }^{*}-\left(\mathbf{v}_{2}{ }^{(1)^{*}} \cdot \nabla\right) \mathbf{v}_{1}{ }^{(1)^{*}}
\end{aligned}
$$

We clarify that the subscripts ' 1 ' and ' 2 ' refer to input fields ' 1 ' and ' 2 ' while the superscripts (1) and (2) refer to first and second order velocity expressions respectively. Using these expressions for $\mathbf{v}^{(1)}$ and $\mathbf{v}^{(2)}$, we substitute into expression (57) for $\mathbf{J}^{(2)}$ :

$$
\mathbf{J}^{(2)}=\left\{\rho_{1}^{(1)} \mathrm{v}_{2}^{(1)}+\rho_{2}^{(1)} \mathrm{v}_{1}^{(1)}+\text { c.c. }\right\}+\rho^{(\mathrm{u})}\left\{\mathbf{v}_{1,2}{ }^{(2)}+\mathbf{v}_{2,1}{ }^{(2)}+\text { c.c. }\right\}
$$

Recalling the definition $\mathbf{J}_{\mathrm{NL}}=\mathbf{J}^{(2)}=\mathbf{J}_{\mathrm{a}}+\mathbf{J}_{\mathrm{a}}{ }^{*}$ :

$$
\mathbf{J}_{\mathbf{a}}=\rho_{1}{ }^{(1)} \mathbf{V}_{2}{ }^{(1)}+\rho_{2}{ }^{(1)} \mathbf{V}_{1}{ }^{(1)}+\rho^{(u)}\left\{\mathbf{v}_{1,2}{ }^{(2)}+\mathbf{v}_{2,1}{ }^{(2)}\right\}
$$

Using equations (62) and (64) :

$$
\begin{array}{r}
\mathbf{J}_{\mathbf{a}}=\rho_{1}^{(1)}\left\{\mathrm{i}(\mathrm{e} / 2 \mathrm{~m})\left(\omega_{2} /\left(\omega_{\mathrm{b}}{ }^{2}-\omega_{2}{ }^{2}\right)\right) \mathbf{E}_{2}\right\}+\rho_{2}{ }^{(1)}\left\{\mathrm{i}(\mathrm{e} / 2 \mathrm{~m})\left(\omega_{1} /\left(\omega_{\mathrm{b}}{ }^{2}-\omega_{1}{ }^{2}\right)\right) \mathbf{E}_{1}\right\}+\rho^{(\mathrm{u})}\left\{\mathbf{v}_{1,2}{ }^{(2)}+\right. \\
\left.\mathbf{v}_{2,1}{ }^{(2)}\right\}(67) \\
\mathbf{J}_{\mathbf{a}}=\rho_{1}^{(1)}\left\{(\mathrm{ie} / 2 \mathrm{~m})\left(\omega_{2} /\left(\omega_{\mathrm{b}}{ }^{2}-\omega_{2}{ }^{2}\right)\right) \mathbf{E}_{2}\right\}+\rho_{2}{ }^{(1)}\left\{(\mathrm{ie} / 2 \mathrm{~m})\left(\omega_{1} /\left(\omega_{\mathrm{b}}{ }^{2}-\omega_{1}{ }^{2}\right)\right) \mathbf{E}_{1}\right\}+\left[\rho^{(\mathrm{u})} / \mathrm{i} \omega_{\text {sum }}\right]\{ \\
\left.(\mathrm{e} /(\mathrm{mc}))\left\{\mathbf{v}_{1}{ }^{(1)} \mathrm{x} \mathbf{B}_{2}+\mathbf{v}_{2}{ }^{(1)} \mathrm{x} \mathbf{B}_{1}\right\}+\left\{\left(\mathbf{v}_{1}{ }^{(1)} \cdot \boldsymbol{\nabla}\right) \mathbf{v}_{2}{ }^{(1)}+\left(\mathbf{v}_{2}{ }^{(1)} \cdot \nabla\right) \mathbf{v}_{1}{ }^{(1)}\right\}\right\}
\end{array}
$$

next from Maxwell's equations :

$$
\nabla \times \mathbf{E}=-1 / \mathrm{cd} \mathbf{B} / \mathrm{dt}=\mathrm{i} \omega / \mathrm{c} \mathbf{B}
$$




\section{Supplemental Information}

so that :

$$
\mathbf{B}=\boldsymbol{\nabla} \mathrm{x} \mathbf{E} \mathrm{c} / \mathrm{i} \omega=\mathbf{k x} \mathbf{E} \mathrm{c} / \omega
$$

Using (68)-(70) :

$$
\begin{aligned}
& \mathbf{J}_{\mathrm{a}}=\rho_{1}{ }^{(1)}\left\{\mathrm{i}(\mathrm{e} / 2 \mathrm{~m})\left(\omega_{2} /\left(\omega_{\mathrm{b}}{ }^{2}-\omega_{2}{ }^{2}\right)\right) \mathbf{E}_{2}\right\}+\rho_{2}{ }^{(1)}\left\{\mathrm{i}(\mathrm{e} / 2 \mathrm{~m})\left(\omega_{1} /\left(\omega_{\mathrm{b}}{ }^{2}-\omega_{1}{ }^{2}\right)\right) \mathbf{E}_{1}\right\}+\left[\rho^{(\mathrm{u})} / \mathrm{i} \omega_{\text {sum }}\right]\{ \\
& \left.(\mathrm{e} / \mathrm{m})\left\{\mathbf{v}_{1}{ }^{(1)} \mathrm{x}\left(\mathbf{k}_{2} \mathrm{x} \mathbf{E}_{2}\right) / \omega_{2}+\mathbf{v}_{2}{ }^{(1)} \mathrm{x}\left(\mathbf{k}_{1} \mathrm{x} \mathbf{E}_{1}\right) / \omega_{1}\right\}+\left\{\left(\mathbf{v}_{1}{ }^{(1)} \cdot \boldsymbol{\nabla}\right) \mathbf{v}_{2}{ }^{(1)}+\left(\mathbf{v}_{2}{ }^{(1)} \cdot \nabla\right) \mathbf{v}_{1}{ }^{(1)}\right\}\right\}
\end{aligned}
$$

Next we use expression (62) for $\mathbf{v}_{\mathbf{j}}^{(1)}$ :

$$
\begin{gathered}
\mathbf{J}_{\mathrm{a}}=\mathrm{i}(\mathrm{e} / 2 \mathrm{~m})\left[\rho_{1}{ }^{(1)} \mathrm{D} \omega_{2} \mathbf{E}_{2}+\rho_{2}{ }^{(1)} \mathrm{D} \omega_{1} \mathbf{E}_{1}\right]+\rho^{(\mathrm{u})}\left[\mathrm{e}^{2} /\left(2 \mathrm{~m}^{2} \omega_{\text {sum }}\right)\right]\left\{\mathrm{D} \omega_{1} \mathbf{E}_{1} \mathrm{x}\left(\mathbf{k}_{2} \mathrm{x} \mathbf{E}_{2}\right) / \omega_{2}+\right. \\
\left.\mathrm{D} \omega_{2} \mathbf{E}_{2} \mathrm{x}\left(\mathbf{k}_{1} \mathrm{x} \mathbf{E}_{1}\right) / \omega_{1}+(\mathrm{i} / 2) \mathrm{D} \omega_{1} \mathrm{D} \omega_{2}\left[\left(\mathbf{E}_{1} \cdot \nabla\right) \mathbf{E}_{2}+\left(\mathbf{E}_{2} \cdot \nabla\right) \mathbf{E}_{1}\right]\right\}
\end{gathered}
$$

Where we have defined :

$$
D \omega_{j} \equiv \omega_{j} /\left(\omega_{b}^{2}-\omega_{j}^{2}\right)
$$

Noting $\left(\mathbf{E}_{1} \cdot \nabla\right) \mathbf{E}_{2}=\mathrm{i}\left(\mathbf{E}_{1} \cdot \mathbf{k}_{2}\right) \mathbf{E}_{2}$ :

$$
\begin{gathered}
\mathbf{J}_{\mathrm{a}}=\mathrm{i}(\mathrm{e} / 2 \mathrm{~m})\left[\mathrm{D} \omega_{2} \rho_{1}{ }^{(1)} \mathbf{E}_{2}+\mathrm{D} \omega_{1} \rho_{2}{ }^{(1)} \mathbf{E}_{1}\right]+\rho^{(\mathrm{u})}\left[\mathrm{e}^{2} /\left(2 \mathrm{~m}^{2} \omega_{\text {sum }}\right)\right]\left\{\mathrm{D} \omega_{1} \mathbf{E}_{1} \mathrm{x}\left(\mathbf{k}_{2} \mathrm{x} \mathbf{E}_{2}\right) / \omega_{2}+\right. \\
\left.\operatorname{D} \omega_{2} \mathbf{E}_{2} \mathrm{x}\left(\mathbf{k}_{1} \mathrm{x} \mathbf{E}_{1}\right) / \omega_{1}-(1 / 2) \mathrm{D} \omega_{1} \mathrm{D} \omega_{2}\left[\left(\mathbf{E}_{1} \cdot \mathbf{k}_{2}\right) \mathbf{E}_{2}+\left(\mathbf{E}_{2} \cdot \mathbf{k}_{1}\right) \mathbf{E}_{1}\right]\right\}
\end{gathered}
$$

We define:

$$
\begin{gathered}
\mathbf{J}_{\text {a Doppler }}(1,2)=\mathrm{i}(\mathrm{e} / 2 \mathrm{~m}) \mathrm{D} \omega_{2} \rho_{1}{ }^{(1)} \mathbf{E}_{2} \\
\mathbf{J}_{\mathrm{a} \text { Displ }}(1,2)=-\rho^{(\mathrm{u})}\left[\mathrm{e}^{2} /\left(4 \mathrm{~m}^{2} \omega_{\text {sum }}\right)\right] \mathrm{D} \omega_{1} \mathrm{D} \omega_{2}\left(\mathbf{E}_{1} \cdot \mathbf{k}_{2}\right) \mathbf{E}_{2} \\
\mathbf{J}_{\text {a Lorentz }}(1,2)=\rho^{(\mathrm{u})}\left[\mathrm{e}^{2} /\left(2 \mathrm{~m}^{2} \omega_{\text {sum }}\right)\right] \mathrm{D} \omega_{1} \mathbf{E}_{1} \mathrm{x}\left(\mathbf{k}_{2} \mathrm{x} \mathbf{E}_{2}\right) / \omega_{2}
\end{gathered}
$$




\section{Supplemental Information}

to rewrite (74) as :

$$
\mathbf{J}_{\mathbf{a}}=\mathbf{J}_{\mathrm{a} \text { Doppler }}(1,2)+\mathbf{J}_{\mathrm{a} \text { Doppler }}(2,1)+\mathbf{J}_{\mathrm{a} \text { Displ }}(1,2)+\mathbf{J}_{\mathrm{a} \text { Displ }}(2,1)+\mathbf{J}_{\mathrm{a} \text { Lorentz }}(1,2)+\mathbf{J}_{\mathrm{a} \text { Lorentz }}(2,1)
$$

Here 'Displ' stands for Displacement. Finally we assign ' 1 '= 'o' for optical and '2'=' $\mathrm{x}$ ' for $\mathrm{x}$-ray to explicitly write the six current terms contributing to $\mathrm{x} / \mathrm{o} \mathrm{SFG}$ :

$$
\begin{gathered}
\mathbf{J}_{\mathrm{a} \text { Doppler }}(\mathrm{o}, \mathrm{x})=\mathrm{i}(\mathrm{e} / 2 \mathrm{~m}) \operatorname{D} \omega_{\mathrm{x}} \rho_{\mathrm{o}}{ }^{(1)} \mathbf{E}_{\mathrm{x}} \\
\mathbf{J}_{\mathrm{a} \text { Doppler }}(\mathrm{x}, \mathrm{o})=\mathrm{i}(\mathrm{e} / 2 \mathrm{~m}) \operatorname{D} \omega_{\mathrm{o}} \rho_{\mathrm{x}}{ }^{(1)} \mathbf{E}_{\mathrm{o}} \\
\mathbf{J}_{\mathrm{a} \text { Displacement }}(\mathrm{o}, \mathrm{x})=-\rho^{(\mathrm{u})}\left[\mathrm{e}^{2} /\left(4 \mathrm{~m}^{2} \omega_{\text {sum }}\right)\right] \operatorname{D} \omega_{\mathrm{o}} \mathrm{D} \omega_{\mathrm{x}}\left(\mathbf{E}_{\mathrm{o}} \cdot \mathbf{k}_{\mathrm{x}}\right) \mathbf{E}_{\mathrm{x}} \\
\mathbf{J}_{\mathrm{a} \text { Displacement }}(\mathrm{x}, \mathrm{o})=-\rho^{(\mathrm{u})}\left[\mathrm{e}^{2} /\left(4 \mathrm{~m}^{2} \omega_{\text {sum }}\right)\right] \mathrm{D} \omega_{\mathrm{x}} \mathrm{D} \omega_{\mathrm{o}}\left(\mathbf{E}_{\mathrm{x}} \cdot \mathbf{k}_{\mathrm{o}}\right) \mathbf{E}_{\mathrm{o}} \\
\mathbf{J}_{\mathrm{a} \text { Lorentz }}(\mathrm{o}, \mathrm{x})=\rho^{(\mathrm{u})}\left[\mathrm{e}^{2} /\left(2 \mathrm{~m}^{2} \omega_{\text {sum }}\right)\right]\left(\mathrm{D} \omega_{\mathrm{o}} / \omega_{\mathrm{x}}\right) \mathbf{E}_{\mathrm{o}} \mathbf{x}\left(\mathbf{k}_{\mathrm{x}} \mathbf{x} \mathbf{E}_{\mathrm{x}}\right) \\
\mathbf{J}_{\mathrm{a} \text { Lorentz }}(\mathrm{x}, \mathrm{o})=\rho^{(\mathrm{u})}\left[\mathrm{e}^{2} /\left(2 \mathrm{~m}^{2} \omega_{\text {sum }}\right)\right]\left(\mathrm{D} \omega_{\mathrm{x}} / \omega_{\mathrm{o}}\right) \mathbf{E}_{\mathrm{x}} \mathbf{x}\left(\mathbf{k}_{\mathrm{o}} \mathbf{x} \mathbf{E}_{\mathrm{o}}\right)
\end{gathered}
$$

We next assume $\mathbf{E}_{\mathrm{o}}$ and $\mathbf{E}_{\mathrm{x}}$ are in the same plane so $\mathbf{E}_{\mathrm{o}} \mathbf{x}\left(\mathbf{k}_{\mathrm{x}} \mathbf{x} \mathbf{E}_{\mathrm{x}}\right)=\mathrm{E}_{\mathrm{o}} \mathrm{E}_{\mathrm{x}} \mathrm{k}_{\mathbf{x}} \mathbf{k}_{\mathrm{o}} /\left|\mathbf{k}_{\mathrm{o}}\right|$ and $\mathbf{E}_{\mathrm{x}} \mathbf{x}\left(\mathbf{k}_{\mathrm{o}} \mathrm{x} \mathbf{E}_{\mathrm{o}}\right)=\mathrm{E}_{\mathrm{o}} \mathrm{E}_{\mathrm{x}} \mathrm{k}_{\mathrm{o}} \mathbf{k}_{\mathrm{x}} /\left|\mathbf{k}_{\mathrm{x}}\right|$. Equations (77) thus becomes :

$$
\begin{gathered}
\mathbf{J}_{\mathrm{a} \text { Doppler }}(\mathrm{o}, \mathrm{x})=\mathrm{i}(\mathrm{e} / 2 \mathrm{~m}) \operatorname{D} \omega_{\mathrm{x}} \rho_{\mathrm{o}}^{(1)} \mathbf{E}_{\mathrm{x}} \\
\mathbf{J}_{\mathrm{a} \text { Doppler }}(\mathrm{x}, \mathrm{o})=\mathrm{i}(\mathrm{e} / 2 \mathrm{~m}) \operatorname{D} \omega_{\mathrm{o}} \rho_{\mathrm{x}}^{(1)} \mathbf{E}_{\mathrm{o}} \\
\mathbf{J}_{\mathrm{a} \text { Displacement }}(\mathrm{o}, \mathrm{x})=-\rho^{(\mathrm{u})}\left[\mathrm{e}^{2} /\left(4 \mathrm{~m}^{2} \omega_{\text {sum }}\right)\right] \mathrm{D} \omega_{\mathrm{o}} \mathrm{D} \omega_{\mathrm{x}}\left(\mathbf{E}_{\mathrm{o}} \cdot \mathbf{k}_{\mathrm{x}}\right) \mathbf{E}_{\mathrm{x}} \\
\mathbf{J}_{\mathrm{a} \text { Displacement }}(\mathrm{x}, \mathrm{o})=-\rho^{(\mathrm{u})}\left[\mathrm{e}^{2} /\left(4 \mathrm{~m}^{2} \omega_{\text {sum }}\right)\right] \mathrm{D} \omega_{\mathrm{x}} \mathrm{D} \omega_{\mathrm{o}}\left(\mathbf{E}_{\mathrm{x}} \cdot \mathbf{k}_{\mathrm{o}}\right) \mathbf{E}_{\mathrm{o}} \\
\mathbf{J}_{\mathrm{a} \text { Lorentz }}(\mathrm{o}, \mathrm{x})=\rho^{(\mathrm{u})}\left[\mathrm{e}^{2} /\left(2 \mathrm{~m}^{2} \omega_{\text {sum }}\right)\right]\left(\mathrm{D} \omega_{\mathrm{o}} / \omega_{\mathrm{x}}\right) \mathrm{E}_{\mathrm{o}} \mathrm{E}_{\mathrm{x}} \mathrm{k}_{\mathrm{x}} \mathbf{k}_{\mathrm{o}} /\left|\mathbf{k}_{\mathrm{o}}\right| \\
\mathbf{J}_{\mathrm{a} \text { Lorentz }}(\mathrm{x}, \mathrm{o})=\rho^{(\mathrm{u})}\left[\mathrm{e}^{2} /\left(2 \mathrm{~m}^{2} \omega_{\text {sum }}\right)\right]\left(\mathrm{D} \omega_{\mathrm{x}} / \omega_{\mathrm{o}}\right) \mathrm{E}_{\mathrm{o}} \mathrm{E}_{\mathrm{x}} \mathrm{k}_{\mathrm{o}} \mathbf{k}_{\mathrm{x}} /\left|\mathbf{k}_{\mathrm{x}}\right|
\end{gathered}
$$




\section{Supplemental Information}

We are interested in the transverse current. Defining $\boldsymbol{\varepsilon}_{\mathrm{SFG}}$ as a unit vector parallel to the polarization direction of the SFG field, the transverse components are written as :

$$
\begin{aligned}
& \mathrm{J}_{\mathrm{a}}^{\mathrm{T}}{ }_{\text {Doppler }}(\mathrm{o}, \mathrm{x})=\mathrm{i}(\mathrm{e} / 2 \mathrm{~m}) \operatorname{D} \omega_{\mathrm{x}} \rho_{\mathrm{o}}^{(1)} \boldsymbol{\varepsilon}_{\mathrm{SFG}} \cdot \mathbf{E}_{\mathrm{x}} \\
& \mathrm{J}_{\mathrm{a}}^{\mathrm{T}} \text { Doppler }(\mathrm{x}, \mathrm{o})=\mathrm{i}(\mathrm{e} / 2 \mathrm{~m}) \operatorname{D} \omega_{\mathrm{o}} \rho_{\mathrm{x}}{ }^{(1)} \boldsymbol{\varepsilon}_{\mathrm{SFG}} \cdot \mathbf{E}_{\mathrm{o}} \\
& \mathrm{J}_{\mathrm{a}}{ }^{\mathrm{T}} \text { Displacement }(\mathrm{o}, \mathrm{x})=-\rho^{(\mathrm{u})}\left[\mathrm{e}^{2} /\left(4 \mathrm{~m}^{2} \omega_{\text {sum }}\right)\right] \mathrm{D} \omega_{\mathrm{o}} \mathrm{D} \omega_{\mathrm{x}}\left(\mathbf{E}_{\mathrm{o}} \cdot \mathbf{k}_{\mathrm{x}}\right) \boldsymbol{\varepsilon}_{\mathrm{SFG}} \cdot \mathbf{E}_{\mathrm{x}} \\
& \mathrm{J}_{\mathrm{a}}^{\mathrm{T}} \text { Displacement }(\mathrm{x}, \mathrm{o})=-\rho^{(\mathrm{u})}\left[\mathrm{e}^{2} /\left(4 \mathrm{~m}^{2} \omega_{\text {sum }}\right)\right] \mathrm{D} \omega_{\mathrm{x}} \mathrm{D} \omega_{\mathrm{o}}\left(\mathbf{E}_{\mathrm{x}} \cdot \mathbf{k}_{\mathrm{o}}\right) \boldsymbol{\varepsilon}_{\mathrm{SFG}} \cdot \mathbf{E}_{\mathrm{o}} \\
& \mathrm{J}_{\mathrm{a}}^{\mathrm{T}} \text { Lorentz }(\mathrm{o}, \mathrm{x})=\rho^{(\mathrm{u})}\left[\mathrm{e}^{2} /\left(2 \mathrm{~m}^{2} \omega_{\text {sum }}\right)\right]\left(D \omega_{\mathrm{o}} / \omega_{\mathrm{x}}\right) \mathrm{E}_{\mathrm{o}} \mathrm{E}_{\mathrm{x}} \mathrm{k}_{\mathrm{x}} \boldsymbol{\varepsilon}_{\mathrm{SFG}} \cdot \mathbf{k}_{\mathrm{o}} /\left|\mathbf{k}_{\mathrm{o}}\right| \\
& \mathrm{J}_{\mathrm{a}}^{\mathrm{T} \text { Lorentz }}(\mathrm{x}, \mathrm{o})=\rho^{(\mathrm{u})}\left[\mathrm{e}^{2} /\left(2 \mathrm{~m}^{2} \omega_{\text {sum }}\right)\right]\left(\mathrm{D} \omega_{\mathrm{x}} / \omega_{\mathbf{0}}\right) \mathrm{E}_{\mathrm{o}} \mathrm{E}_{\mathrm{x}} \mathrm{k}_{\mathbf{0}} \boldsymbol{\varepsilon}_{\mathrm{SFG}} \cdot \mathbf{k}_{\mathrm{x}} /\left|\mathbf{k}_{\mathrm{x}}\right|
\end{aligned}
$$

Finally, the nonlinear source term is twice the $\mathbf{J}_{\mathrm{a}}$ terms :

$$
\begin{array}{r}
\mathrm{J}^{\mathrm{T}}{ }_{\mathrm{NL}}=2\left\{\mathrm{~J}_{\mathrm{a}}{ }^{\mathrm{T}} \text { Doppler }(\mathrm{o}, \mathrm{X})+\mathrm{J}_{\mathrm{a}}{ }^{\mathrm{T}} \text { Doppler }(\mathrm{x}, \mathrm{o})+\mathrm{J}_{\mathrm{a}}{ }^{\mathrm{T}} \text { Displacement }(\mathrm{o}, \mathrm{x})+\mathrm{J}_{\mathrm{a}}{ }^{\mathrm{T}} \text { Displacement }(\mathrm{x}, \mathrm{o})+\right. \\
\left.\mathrm{J}_{\mathrm{a}}{ }^{\mathrm{T}} \text { Lorentz }(\mathrm{o}, \mathrm{x})+\mathrm{J}_{\mathrm{a}}{ }^{\mathrm{T}}{ }_{\text {Lorentz }}(\mathrm{x}, \mathrm{o})\right\}
\end{array}
$$

\section{X-ray/optical SFG at other light sources}

We consider $\mathrm{x}$-ray/optical SFG at different $\mathrm{x}$-ray light sources by comparing the number of useful source photons. We consider three sources: an existing x-ray FEL (Linac Coherent Light Source, LCLS), an existing third generation synchrotron (Advanced Photon Source, APS), and a proposed light source (Energy Recovery Linac, ERL). The source parameters are taken from [42] and [43].

We first consider the peak brightness $\left(\mathrm{B}_{\mathrm{P}}\right)$, in units of Photons $/ \mathrm{s}^{*} \mathrm{~mm}^{2 *} \operatorname{mrad}^{2} * 0.1 \%$ bandwidth for one pulse. We take the APS, ERL, and LCLS peak brightness to be $\mathrm{B}_{\mathrm{P}, \mathrm{APS}}=3 \times 10^{22}$, $\mathrm{B}_{\mathrm{P}, \mathrm{ERL}}=3 \times 10^{25}$, and $\mathrm{B}_{\mathrm{P}, \mathrm{LCLS}}=10^{32}$. We next multiply the peak brightness by the pulse duration $(\tau)$, the beam area (A), and the 


\section{Supplemental Information}

out-of-diffraction-plane beam divergence $\left(\delta \theta_{\text {out }}\right)$ to obtain a metric, $M_{A}$, defined as the number of photons per mrad per $0.1 \%$ bandwidth in one pulse. The source parameters are taken as :

$$
\begin{aligned}
& \tau_{\mathrm{LCLS}}=200 \mathrm{fs} ; \tau_{\mathrm{ERL}}=300 \mathrm{fs} ; \tau_{\mathrm{APS}}=80 \mathrm{ps} \\
& \mathrm{A}_{\mathrm{LCLS}}=80 \times 80 \mathrm{um}^{2} ; \mathrm{A}_{\mathrm{ERL}}=100 \times 100 \mathrm{um}^{2} ; \mathrm{A}_{\mathrm{APS}}=840 \times 50 \mathrm{um}^{2} \\
& \delta \theta_{\text {out }, \mathrm{LCLS}}=1 \mu \mathrm{r} ; \quad \delta \theta_{\text {out }, \mathrm{ERL}}=9 \mu \mathrm{r} ; \quad \delta \theta_{\text {out }, \mathrm{APS}}=55 \mu \mathrm{r}
\end{aligned}
$$

The ' $\mathrm{M}_{\mathrm{A}}$ " values are :

$$
\begin{aligned}
& \mathrm{M}_{\mathrm{A}, \mathrm{LCLS}}=1.6 \times 10^{5} \\
& \mathrm{M}_{\mathrm{A}, \mathrm{APS}}=6.8 \\
& \mathrm{M}_{\mathrm{A}, \mathrm{ERL}}=1
\end{aligned}
$$

where relative units have been used; that is, $M_{A, E R L}$ has been assigned the value ' 1 ' and $\mathrm{M}_{\mathrm{A}, \mathrm{APS}}, \mathrm{M}_{\mathrm{A}, \mathrm{LCLS}}$ are relative to this value. We note that the total number of useful source photons per pulse for APS is somewhat higher than for the ERL, and both are small compared to LCLS.

Finally, we multiply by the laser repetition rate to determine the number of useful source photons per second. First we consider the exact apparatus of our LCLS experiment; this means we choose a $1 \mathrm{KHz}$ repetition rate optical laser and assume that a two-bounce $\mathrm{Si}$ (111) monchromator 'conditions' the input x-ray beam before it strikes the diamond sample. We note that all three sources are broad in energy relative to the monchromator energy bandpass $(\sim 1 \mathrm{eV}$ at $8 \mathrm{keV})$ so that the monochromator bandpass determines the x-ray bandwidth incident on the sample. Further, while the three sources have different in-diffraction-plane divergence, we calculate that the differences $\left(\delta \theta_{\text {in,APS }}\right.$ $=16 \mu \mathrm{rad}, \delta \theta_{\text {in,ERL }}=9 \mu \mathrm{rad}, \delta \theta_{\text {in,LCLS }}=1 \mu \mathrm{rad}$; from [42] and [43]) lead to relatively 


\section{Supplemental Information}

small variation in the SFG efficiency (relative efficiencies of $\eta_{\mathrm{APS}}=1, \eta_{\mathrm{ERL}}=1.3$, $\left.\eta_{\mathrm{LCLS}}=1.4\right)$.

The LCLS currently runs at a repetition rate of $120 \mathrm{~Hz}$, limited by the x-ray laser itself ( $60 \mathrm{~Hz}$ at the time of our experiment). The APS and ERL experiments are limited by the repetition rate of the optical laser $(1 \mathrm{KHz})$. Defining metric $\mathrm{M}_{\mathrm{B}}$ as the number of useful source photons per second per mrad per $0.1 \%$ bandwidth (i.e. $\mathrm{M}_{\mathrm{B}}=\mathrm{M}_{\mathrm{A}}$ times the repetition rate) we obtain (again specifying in relative units) :

$$
\begin{aligned}
& \mathrm{M}_{\mathrm{B}, \mathrm{LCLS}}=2770 \\
& \mathrm{M}_{\mathrm{B}, \mathrm{APS}}=1 \\
& \mathrm{M}_{\mathrm{B}, \mathrm{ERL}}=0.15
\end{aligned}
$$

Accounting for the efficiency factors above $\left(\eta_{\mathrm{APS}}=1, \eta_{\mathrm{ERL}}=1.3, \eta_{\mathrm{LCLS}}=1.4\right)$, we estimate that the APS is somewhat better suited to $\mathrm{x} / \mathrm{o}$ mixing than is the ERL and that with an identical experimental apparatus the APS signal rate is approximately a factor of 4000 smaller than the LCLS signal rate. In principle this deficit could be overcome by taking advantage of the much higher repetition rate of the APS (and ERL) as compared to the LCLS. By using a high repetition rate $(1 \mathrm{MHz})$ laser system rather than the $1 \mathrm{KHz}$ system of the current experiment, it may be possible to approach count rates comparable to the LCLS signal rate. The APS pulse duration is approximately $100 \mathrm{ps}$. Assuming an optical pulse with the same duration, an optical intensity of $10^{10} \mathrm{~W} / \mathrm{cm}^{2}$ can be obtained with an optical pulse energy of $10 \mu \mathrm{J}$ and a focal spot diameter of $30 \mu \mathrm{m}$ (optical and $\mathrm{x}$ ray). The longer optical pulse required at the APS ( $\sim 100 \mathrm{ps}$ vs $\sim 100 \mathrm{fs}$ ) combined with the higher average heat load on the sample may lead to sample damage. 


\section{Supplemental Information}

\section{References}

[41] Dawson, B., The Covalent Bond in Diamond. Proc. R. Soc. Lond. A 298, 264-288 (1967).

[42]https://slacportal.slac.stanford.edu/sites/lclscore_public/Accelerator_Physics_Publis hed_Documents/LCLS-parameters.pdf

[43] CHESS Technical Memo 01-002,

http://erl.chess.cornell.edu/papers/2001/ERLPub01_4.pdf 